\title{
Statistics of the MLE and Approximate Upper and Lower Bounds-Part I: Application to TOA Estimation
}

\author{
Achraf Mallat, Member, IEEE, Sinan Gezici, Senior Member, IEEE, Davide Dardari, Senior Member, IEEE, \\ Christophe Craeye, Senior Member, IEEE, and Luc Vandendorpe, Fellow, IEEE
}

\begin{abstract}
In nonlinear deterministic parameter estimation, the maximum likelihood estimator (MLE) is unable to attain the Cramér-Rao lower bound at low and medium signal-to-noise ratios (SNRs) due the threshold and ambiguity phenomena. In order to evaluate the achieved mean-squared error (MSE) at those SNR levels, we propose new MSE approximations (MSEA) and an approximate upper bound by using the method of interval estimation (MIE). The mean and the distribution of the MLE are approximated as well. The MIE consists in splitting the $a$ priori domain of the unknown parameter into intervals and computing the statistics of the estimator in each interval. Also, we derive an approximate lower bound (ALB) based on the Taylor series expansion of noise and an ALB family by employing the binary detection principle. The accuracy of the proposed MSEAs and the tightness of the derived approximate bounds are validated by considering the example of time-of-arrival estimation.
\end{abstract}

Index Terms - Nonlinear estimation, threshold and ambiguity phenomena, maximum likelihood estimator, mean-squared error, upper and lowers bounds, time-of-arrival.

\section{INTRODUCTION}

$\mathbf{N}$ ONLINEAR estimation of deterministic parameters suffers from the threshold effect [2]-[11]. This effect means that for a signal-to-noise ratio (SNR) above a given threshold, estimation can achieve the Cramer-Rao lower bound (CRLB), whereas for SNRs lower than that threshold, estimation deteriorates drastically until the estimate becomes uniformly distributed in the a priori domain of the unknown parameter.

As depicted in Fig. 1(a), the SNR axis can be split into three regions according to the achieved mean-squared-error (MSE):

Manuscript received October 20, 2013; revised April 07, 2014 and August 15, 2014; accepted August 26, 2014. Date of publication September 08, 2014; date of current version October 07, 2014. The associate editor coordinating the review of this manuscript and approving it for publication was Dr. Petr Tichavsky. This work has been supported in part by the Belgian IAP network Bestcom funded by Belspo, the PEGASO project funded by the Walloon region Skywin pole, the FNRS, and the European Commission in the framework of the FP7 Network of Excellence in Wireless COMmunications NEWCOM \# (Contract no. 318306). S. Gezici's research was supported in part by the Young Scientists Award Programme of Turkish Academy of Sciences (TUBA-GEBIP 2013).

A. Mallat, C. Craeye, and L. Vandendorpe are with the ICTEAM Institute, Université Catholique de Louvain, Louvain-la-Neuve 1348, Belgium (e-mail: Achraf.Mallat@uclouvain.be; Christophe.Craeye@uclouvain.be; Luc.Vandendorpe@uclouvain.be).

S. Gezici is with the Department of Electrical and Electronics Engineering, Bilkent University, Ankara 06800, Turkey (e-mail: gezici@ee.bilkent.edu.tr).

D. Dardari is with DEI, CNIT, University of Bologna, Bologna 40126, Italy (e-mail: davide.dardari@unibo.it).

Color versions of one or more of the figures in this paper are available online at http://ieeexplore.ieee.org.

Digital Object Identifier 10.1109/TSP.2014.2355771

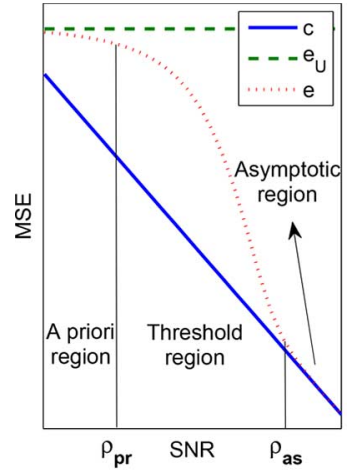

(a)

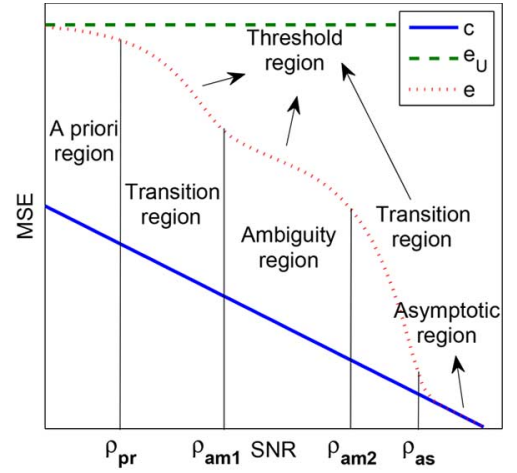

(b)
Fig. 1. SNR regions: (a) a priori, threshold and asymptotic regions for non-oscillating ACRs and (b) a priori, ambiguity and asymptotic regions for oscillating ACRs ( $c$ : CRLB, $e_{U}$ : MSE of uniform distribution in the a priori domain, $e$ : achievable MSE, $\rho_{p r}, \rho_{a m 1}, \rho_{a m 2}, \rho_{a s}:$ a priori, begin-ambiguity, end-ambiguity and asymptotic thresholds).

1) A priori region: Region in which the estimate is uniformly distributed in the a priori domain of the unknown parameter (region of low SNRs).

2) Threshold region: Region of transition between the a priori and asymptotic regions (region of medium SNRs).

3) Asymptotic region: Region in which the CRLB is achieved (region of high SNRs).

In addition, if the autocorrelation (ACR) of the signal carrying the information about the unknown parameter is oscillating, then estimation will be affected by the ambiguity phenomenon ([12], pp. 119) and a new region will appear so the SNR axis can be split, as shown Fig. 1(b), into five regions:

1) A priori region.

2) A priori-ambiguity transition region.

3) Ambiguity region.

4) Ambiguity-asymptotic transition region.

5) Asymptotic region.

The MSE achieved in the ambiguity region is determined by the envelope of the ACR. In Figs. 1(a) and (b), we denote by $\rho_{p r}$, $\rho_{a m 1}, \rho_{a m 2}$ and $\rho_{a s}$ the a priori, begin-ambiguity, end-ambiguity and asymptotic thresholds delimiting the different regions. The CRLB is not achieved asymptotically unless the used estimator is asymptotically efficient. For example, the maximum likelihood estimator (MLE) in [13] (with deterministic signals) asymptotically achieves the CRLB whereas the MLE in [14] (with random signals and finite snapshots) and the Capon algorithm in [15] do not achieve it. 
The exact evaluation of the statistics, in the threshold region, of some estimators such as the MLE has been considered as a prohibitive task. Many lower bounds (LB) have been derived for both deterministic and Bayesian (when the unknown parameter follows a given a priori distribution) parameters in order to be used as benchmarks and to describe the behavior of the MSE in the threshold region [16]. Some upper bounds (UB) have also been derived like the Seidman UB [17]. It will suffice to mention here [16], [18] the Cramer-Rao, Bhattacharyya, Chapman-Robbins, Barankin and Abel deterministic LBs, the Cramer-Rao, Bhattacharyya, Bobrovsky-MayerWolf-Zakai, Bobrovsky-Zakai, and Weiss-Weinstein Bayesian LBs, the Ziv-Zakai Bayesian LB (ZZLB) [2] with its improved versions: Bellini-Tartara [4], Chazan-Ziv-Zakai [19], Weinstein [20] (approximation of Bellini-Tartara), and Bell-Steinberg-Ephraim-VanTrees [21] (generalization of Ziv-Zakai and Bellini-Tartara), and the Reuven-Messer LB [22] for problems of simultaneously deterministic and Bayesian parameters.

The CRLB [23] gives the minimum MSE achievable by an unbiased estimator. However, it can be very optimistic at low and moderate SNRs when the estimator is not efficient; furthermore, it is unable to model the threshold and ambiguity regions. The Barankin LB (BLB) [24] gives the greatest LB of an unbiased estimator. However, its general form is not easy to compute for most interesting problems. A useful form of this bound, which is much tighter than the CRLB, is derived in [25] and generalized to vector cases in [26]. As shown in [3], [27], as well as in our numerical results in Section VII, the bound in [25] detects the asymptotic region much below the true one. Some applications of the BLB can be found in [5], [8], [9], [28], [29].

The Bayesian ZZLB family [2], [4], [19]-[21] is based on the minimum probability of error of a binary detection problem. The ZZLBs are very tight; they detect the ambiguity region roughly and the asymptotic region accurately. Some applications of the ZZLBs, discussions and comparison to other bounds can be found in [10]-[12], [27], [30]-[35].

In ([36], pp. 627-637), Wozencraft considered time-of-arrival (TOA) estimation with cardinal sine waveforms and employed the method of interval estimation (MIE) to approximate the MSE of the MLE. The MIE ([18], pp. 58-62) consists in splitting the a priori domain of the unknown parameter into intervals and computing the probability that the estimate falls in a given interval, and the estimator mean and variance in each interval. According to [18], [37], the MIE was first used in [38], [39] before Wozencraft [36] and others introduced some modifications later. The approach in [36] is imitated in [18], [37], [40], [41] for frequency estimation and in [42] for angle-of-arrival (AOA) estimation. The ACRs in [15], [18], [36], [37], [40]-[42] have the special shape of a cardinal sine (oscillating baseband with the mainlobe twice wider than the sidelobes); this limitation makes their approach inapplicable on other shapes. In [1], McAulay considered TOA estimation with carrier-modulated pulses (oscillating passband ACRs) and used the MIE to derive an approximate UB (AUB) ${ }^{1}$; the approach of McAulay can be applied to any oscillating ACR. Indeed, it is followed (indepen- dently apparently) in [15], [43], [44] for AOA estimation and in [41] (for frequency estimation as mentioned above) where it is compared to Wozencraft's approach. The ACR considered in [43], [44] has an arbitrary oscillating baseband shape (due to the use of non-regular arrays), meaning that it looks like a cardinal sine but with some strong sidelobes arbitrarily located. The MSEAs based on Wozencraft's approach are very accurate and the AUBs using McAulay's approach are very tight in the asymptotic and threshold regions. Both approaches can be used to determine accurately the asymptotic region. Various estimators are considered in the previously cited references. More technical details about the MIE are given in Section IV.

In this paper, we consider the estimation of a scalar deterministic parameter. Compared with the presented state-of-the-art, our work makes the following main contributions:

- We employ the MIE to propose new approximations (rather than AUBs) of the MSE achieved by the MLE and a very tight AUB. The proposed MSEAs are highly accurate. One of these approximations is expressed as the sum of two terms. The mean and the probability density function (PDF) of the MLE are approximated as well. More details about the novelties with regards to the MIE are given in Sections IV and V.

- We derive an approximate LB (ALB) tighter than the CRLB based on the second order Taylor series expansion of the noise.

- We utilize the binary detection principle to derive some ALBs; the obtained bounds are very tight.

The theoretical results presented in this paper are applicable to any estimation problem satisfying the system model introduced in Section II. In order to illustrate the accurateness of the proposed MSEAs and the tightness of the derived bounds, we consider the example of TOA estimation with baseband and passband pulses.

The materials presented in this paper compose the first part of our work divided in two parts (see [45]).

The rest of the paper is organized as follows. In Section II we introduce our system model. In Section III we describe the threshold and ambiguity phenomena. In Section IV we deal with the MIE. In Section V we propose an AUB and an MSEA. In Section VI we derive some ALBs. In Section VII we consider the example of TOA estimation and discuss the obtained numerical results.

\section{SYSTEM MODEL}

In this section we consider the general estimation problem of a deterministic scalar parameter (Section II.A) and the particular case of TOA estimation (Section II.B).

\section{A. Deterministic Scalar Parameter Estimation}

Let $\Theta$ be a deterministic unknown parameter with $D_{\Theta}=$ $\left[\Theta_{1}, \Theta_{2}\right]$ denoting its a priori domain. We can write the $i$ th, $(i=1, \ldots, I)$ observation as:

$$
r_{i}(t)=\alpha s_{i}(t ; \Theta)+\tilde{w}_{i}(t)
$$

\footnotetext{
${ }^{1}$ The derived magnitude is referred as "bound" because it is greater than the MSE, and as "approximate" because an approximation is performed to obtain it; the terminology "approximate bound" is adopted in our paper as well.
} 
where $s_{i}(t ; \Theta)$ is the $i$ th useful signal carrying the information on $\Theta, \alpha$ is a known positive gain, and $\tilde{w}_{i}(t)$ is an additive white Gaussian noise (AWGN) with two-sided power spectral density (PSD) of $\frac{N_{0}}{2} ; \tilde{w}_{1}(t), \ldots, \tilde{w}_{I}(t)$ are independent.

Denote by $E_{x}(\theta)=\sum_{i=1}^{I} \int_{-\infty}^{+\infty} x_{i}^{2}(t ; \theta) d t$ the sum of the energies of $x_{1}(t ; \theta), \ldots, x_{I}(t ; \theta)$, by $\dot{x}$ and $\ddot{x}$ the first and second derivatives of $x$ w.r.t. $\theta$, and by $\mathbb{E}, \Re$ and $\mathbb{P}$ the expectation, real part and probability operators respectively. From (1) we can write the log-likelihood function of $\Theta$ as:

$$
\Lambda(\theta)=-\frac{1}{N_{0}}\left[E_{r}+\alpha^{2} E_{s}(\theta)-2 \alpha X_{s, r}(\theta)\right]
$$

where $\theta \in D_{\Theta}$ denotes a variable associated with $\Theta$, and

$$
X_{s, r}(\theta)=\sum_{i=1}^{I} \int_{-\infty}^{+\infty} s_{i}(t ; \theta) r_{i}(t) d t=\alpha R_{s}(\theta, \Theta)+w(\theta)
$$

is the crosscorrelation (CCR) with respect to (w.r.t.) $\theta$, with

$$
R_{s}\left(\theta, \theta^{\prime}\right)=\sum_{i=1}^{I} \int_{-\infty}^{+\infty} s_{i}(t ; \theta) s_{i}\left(t ; \theta^{\prime}\right) d t
$$

denoting the ACR w.r.t. $\left(\theta, \theta^{\prime}\right)$ and

$$
w(\theta)=\sum_{i=1}^{I} \int_{-\infty}^{+\infty} s_{i}(t ; \theta) \tilde{w}_{i}(t) d t
$$

being a colored zero-mean Gaussian noise of covariance

$$
C_{w}\left(\theta, \theta^{\prime}\right)=\sum_{i=1}^{I} \mathbb{E}\left\{w_{i}(\theta) w_{i}\left(\theta^{\prime}\right)\right\}=\frac{N_{0}}{2} R_{s}\left(\theta, \theta^{\prime}\right) .
$$

1) MLE, $C R L B$ and Envelope $C R L B$ : By assuming $E_{s}(\theta)=$ $E_{s}$ in (2), that is, $E_{s}(\theta)$ is independent of $\theta$ (true for many estimation problems such as the ones mentioned at the end of Section II.A), we can write the MLE $\hat{\Theta}$ and the CRLB $c(\Theta)$ of $\Theta$ as ([23], pp. 39):

$$
\begin{aligned}
\hat{\Theta} & =\underset{\theta \in D_{\Theta}}{\operatorname{argmax}} X_{s, r}(\theta) \\
c(\Theta) & =\frac{-1}{\mathbb{E}\left\{\left.\ddot{\Lambda}(\theta)\right|_{\theta=\Theta}\right\}}=\frac{-N_{0} / 2}{\alpha^{2} \ddot{R}_{s}(\Theta, \Theta)}=\frac{1}{\rho \beta_{s}^{2}(\Theta)}
\end{aligned}
$$

where

$$
\begin{aligned}
\rho & =\frac{\alpha^{2} E_{s}}{N_{0} / 2} \\
\beta_{s}^{2}(\Theta) & =-\frac{\ddot{R}_{s}(\Theta, \Theta)}{E_{s}}
\end{aligned}
$$

denote the SNR and the normalized curvature of $R_{s}(\theta, \Theta)$ at $\theta=\Theta$ respectively. Unlike $E_{s}(\Theta), \ddot{R}_{s}(\Theta, \Theta)$ may depend on $\Theta$ (e.g., AOA estimation [46]). The CRLB in (8) is inversely proportional to the curvature of the ACR at $\theta=\Theta$. Sometimes $R_{s}(\theta, \Theta)$ is oscillating w.r.t. $\theta$. Then, if the SNR is sufficiently high (resp. relatively low) the maximum of the CCR in (3) will fall around the global maximum (resp. the local maxima) of $R_{s}(\theta, \Theta)$ and the MLE in (7) will (resp. will not) achieve the CRLB. We will see in Section VII that the MSE achieved at medium SNRs is inversely proportional to the curvature of the envelope of the ACR instead of the curvature of the ACR itself. To characterize this phenomenon known as "ambiguity" [47] we will define below the envelope CRLB (ECRLB).

Denote by $f$ the frequency ${ }^{2}$ relative to $\theta$ and define the Fourier transform (FT), the mean frequency and the complex envelope w.r.t. $f_{c}(\Theta)$ of $R_{s}(\theta, \Theta)$ respectively by

$$
\begin{aligned}
\mathcal{F}_{R_{s}}(f) & =\int_{\Theta_{1}}^{\Theta_{2}} R_{s}(\theta, \Theta) e^{-j 2 \pi f(\theta-\Theta)} d \theta \\
f_{c}(\Theta) & =\frac{\int_{0}^{+\infty} f \Re\left\{\mathcal{F}_{R_{s}}(f)\right\} d f}{\int_{0}^{+\infty} \Re\left\{\mathcal{F}_{R_{s}}(f)\right\} d f} \\
R_{s}(\theta, \Theta) & =\Re\left\{e^{j 2 \pi(\theta-\Theta) f_{c}(\Theta)} e_{R_{s}}(\theta, \Theta)\right\} .
\end{aligned}
$$

In Appendix A we show that:

$$
-\ddot{R}_{s}(\Theta, \Theta)=-\Re\left\{\ddot{e}_{R_{s}}(\Theta, \Theta)\right\}+4 \pi^{2} f_{c}^{2}(\Theta) E_{s} .
$$

Now, we define the ECRLB as:

$$
c_{e}(\Theta)=-\frac{N_{0} / 2}{\alpha^{2} \Re\left\{\ddot{e}_{R_{s}}(\Theta, \Theta)\right\}}=\frac{1}{\rho \beta_{e}^{2}(\Theta)}
$$

where

$$
\beta_{e}^{2}(\Theta)=-\frac{\Re\left\{\ddot{e}_{R_{s}}(\Theta, \Theta)\right\}}{E_{s}}
$$

denotes the normalized curvature of $e_{R_{s}}(\theta, \Theta)$ at $\theta=\Theta$. From (10), (14) and (16), we have:

$$
\beta_{s}^{2}(\Theta)=\beta_{e}^{2}(\Theta)+4 \pi^{2} f_{c}^{2}(\Theta) .
$$

2) $B L B$ : The BLB can be written as [25]:

$$
c_{B}=(\underline{\Theta}-\Theta)^{T} D^{-1}(\underline{\Theta}-\Theta)
$$

where

$$
\begin{aligned}
\underline{\Theta} & =\left(\theta_{n_{1}} \cdots \theta_{-1} 1+\Theta \theta_{1} \cdots \theta_{n_{N}}\right)^{T} \\
D & =\left.\left(d_{i, j}\right)\right|_{i, j=n_{1}, \ldots, n_{N}}
\end{aligned}
$$

with $\theta_{n_{1}}, \ldots, \theta_{n_{N}}\left(n_{1} \leq 0, n_{N} \geq 0, \theta_{0}=\Theta\right)$ denoting $N$ testpoints in the a priori domain of $\Theta$, and ${ }^{3}$

$$
\begin{aligned}
d_{0,0} & =\frac{\alpha^{2} E_{\dot{s}}(\Theta)}{N_{0} / 2}=\frac{1}{c(\Theta)} \\
d_{0, i \neq 0} & =d_{i, 0}=\frac{\alpha^{2}}{N_{0} / 2}\left[\dot{R}_{s}\left(\Theta, \theta_{i}\right)-\dot{R}_{s}(\Theta, \Theta)\right] \\
d_{i \neq 0, j \neq 0} & =\frac{\alpha^{2}}{N_{0} / 2}\left[R_{s}\left(\theta_{i}, \theta_{j}\right)-R_{s}\left(\theta_{i}, \Theta\right)-R_{s}\left(\theta_{j}, \Theta\right)+E_{s}\right] .
\end{aligned}
$$

3) Maximum MSE: The maximum MSE

$$
e_{U}=\sigma_{U}^{2}+\left(\Theta-\mu_{U}\right)^{2}
$$

${ }^{2} \mathrm{E} . \mathrm{g}, f$ is in seconds (resp. Hz) for frequency (resp. TOA) estimation.

${ }^{3}$ We can show that $E_{\dot{s}}(\theta)=-\ddot{R}_{s}(\theta, \Theta)$ if $E_{s}(\theta)$ is independent from $\theta$. 


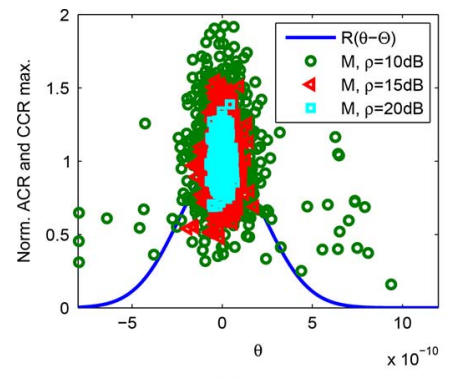

(a)

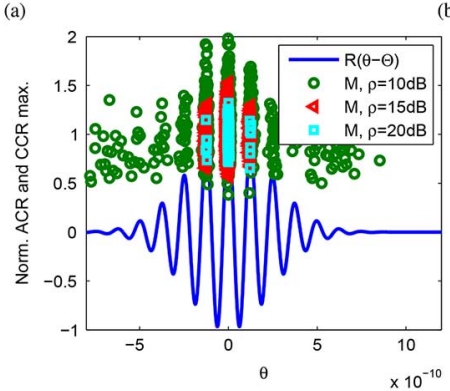

(c)

Fig. 2. Normalized ACR $R(\theta-\Theta)$ and 1000 realizations of $M[\hat{\Theta}, X(\hat{\Theta})]$ per SNR $(\rho=10,15$ and $20 \mathrm{~dB})$; Gaussian pulse modulated by $f_{c}, \Theta=0 \mathrm{~ns}$, $T_{w}=0.6 \mathrm{~ns}, D_{\Theta}=[-1.5,1.5] T_{w}$. (a) $f_{c}=0 \mathrm{GHz}$. (b) $f_{c}=4 \mathrm{GHz}$. (c) $f_{c}=8 \mathrm{GHz}$.

TABLE I

CRLB SQRT $\sqrt{c}$ (PS), SIMULATED RMSE $\sqrt{e_{S}}$ (PS), RMSE TO CRLB SQRT RATIO $\sqrt{\frac{e_{S}}{c}}$, AND NUMBER $\left(N_{0}, N_{1}\right)$ OF THE $M$ SAmples Falling ARound the Maxima Number 0 and 1, FOR $f_{c}=0,4$ AND $8 \mathrm{GHz}$, AND $\rho=10,15$ AND $20 \mathrm{~dB}$

\begin{tabular}{|c|c||c|c|c|c|c|}
\hline$f_{c}$ & $\rho$ & $\sqrt{c}$ & $\sqrt{e_{S}}$ & $\sqrt{\frac{e_{S}}{c}}$ & $N_{0}$ & $N_{1}$ \\
\hline \hline \multirow{4}{*}{0} & 10 & 76 & 123 & 1.61 & 1000 & 0 \\
& 15 & 43 & 46 & 1.10 & 1000 & 0 \\
& 20 & 24 & 24 & 1.01 & 1000 & 0 \\
\hline \multirow{4}{*}{4} & 10 & 12 & 196 & 15.81 & 773 & 59 \\
& 15 & 7 & 31 & 4.47 & 985 & 8 \\
& 20 & 4 & 4 & 1.01 & 1000 & 0 \\
\hline \multirow{4}{*}{8} & 10 & 6.3 & 198 & 31.56 & 481 & 199 \\
& 15 & 3.5 & 50 & 14.35 & 838 & 75 \\
& 20 & 2 & 14 & 7.14 & 987 & 7 \\
\hline
\end{tabular}

with $\mu_{U}=\frac{\Theta_{1}+\Theta_{2}}{2}$ and $\sigma_{U}^{2}=\frac{\left(\Theta_{2}-\Theta_{1}\right)^{2}}{12}$ is achieved when the estimator becomes uniformly distributed in $D_{\Theta}$ [30], [34].

The system model considered in this subsection is satisfied for various estimation problems such as TOA, AOA, phase, frequency and velocity estimation. Therefore, the theoretical results presented in this paper are valid for the different mentioned parameters. TOA is just considered as an example to validate the accurateness and the tightness of our MSEAs and upper and lowers bounds.

\section{B. Example: TOA Estimation}

With TOA estimation based on one observation $(I=1)$, $s_{1}(t ; \Theta)$ in $(1)$ becomes $s_{1}(t ; \Theta)=s(t-\Theta)$ where $s(t)$ denotes the transmitted signal and $\Theta$ represents the delay introduced by the channel. Accordingly, we can write the ACR in (4) as
$R_{s}\left(\theta, \theta^{\prime}\right)=R_{s}\left(\theta-\theta^{\prime}\right)$ where $R_{s}(\theta)=\int_{-\infty}^{+\infty} s(t+\theta) s(t) d t$, and the CCR in (3) as:

$$
X_{s, r}(\theta)=\alpha R_{s}(\theta-\Theta)+w(\theta) .
$$

The CRLB $c(\Theta)$ in (8), ECRLB $c_{e}(\Theta)$ in (15), mean frequency $f_{c}(\Theta)$ in (12), normalized curvatures $\beta_{s}^{2}(\Theta)$ in $(10)$ and $\beta_{e}^{2}(\Theta)$ in (16) become now all independent of $\Theta$. Furthermore, $\beta_{s}^{2}$ and $\beta_{e}^{2}$ denote now the mean quadratic bandwidth (MQBW) and the envelope MQBW (EMQBW) of $s(t)$ respectively.

The CRLB in (8) is much smaller than the ECRLB in (15) because the MQBW in (17) is much larger than the EMQBW in (16). In fact, for a signal occupying the whole band from 3.1 to $10.6 \mathrm{GHz}^{4}\left(f_{c}=6.85 \mathrm{GHz}\right.$, bandwidth $\left.B=7.5 \mathrm{GHz}\right)$, we ob$\operatorname{tain} \beta_{e}^{2}=\frac{\pi^{2} B^{2}}{3} \approx 185 \mathrm{GHz}^{2}, 4 \pi^{2} f_{c}^{2} \approx 10 \beta_{e}^{2}, \beta_{s}^{2} \approx 11 \beta_{e}^{2}$ and $c \approx \frac{c_{e}}{11}$. Therefore, the estimation performance seriously deteriorates at relatively low SNRs when the ECRLB is achieved instead of the CRLB due to ambiguity.

\section{Threshold AND Ambiguity Phenomena}

In this section we explain the physical origin of the threshold and ambiguity phenomena by considering TOA estimation with UWB pulses 5 as an example. The transmitted signal

$$
s(t)=2 \sqrt{\frac{E_{s}}{T_{w}}} e^{-2 \pi \frac{t^{2}}{T_{w}^{2}}} \cos \left(2 \pi f_{c} t\right)
$$

is a Gaussian pulse of width $T_{w}$ modulated by a carrier $f_{c}$. We consider three values of $f_{c}\left(f_{c}=0,4\right.$ and $\left.8 \mathrm{GHz}\right)$ and three values of the SNR ( $\rho=10,15$ and $20 \mathrm{~dB})$ per considered $f_{c}$. We take $\Theta=0, T_{w}=0.6 \mathrm{~ns}$, and $D_{\Theta}=[-1.5,1.5] T_{w}$.

In Figs. 2(a)-(c) we show the normalized ACR $R(\theta-\Theta)=$ $\frac{R_{s}(\theta-\Theta)}{E_{s}}$ for $f_{c}=0$ (baseband pulse), 4 and $8 \mathrm{GHz}$ (passband pulses) respectively, and 1000 realizations per SNR of the maximum $M[\hat{\Theta}, X(\hat{\Theta})]$ of the normalized CCR $X(\theta)=\frac{X_{s, r}(\theta)}{\alpha E_{s}}$. Denote by $N_{n},\left(n=n_{1}, \ldots, n_{N}\right),(N$ is the number of local maxima in $\left.D_{\Theta}\right),\left(n_{1}<0, n_{N}>0\right),(n=0$ corresponds to the global maximum) the number of samples of $M$ falling around the $n$th local maximum (i.e., between the two local minima adjacent to that maximum) of $R(\theta-\Theta)$. In Table I, we show w.r.t. $f_{c}$ and $\rho$ the number of samples falling around the maxima number 0 and 1 , the CRLB square root (SQRT) $\sqrt{c}$ of $\Theta$, the root MSE (RMSE) $\sqrt{e_{S}}$ obtained by simulation and the RMSE to CRLB SQRT ratio $\sqrt{\frac{e_{S}}{c}}$.

Consider first the baseband pulse. We can see in Fig. 2(a) that the samples of $M$ are very close to the maximum of $R(\theta-\Theta)$ for $\rho=20 \mathrm{~dB}$, and they start to spread progressively along $R(\theta-\Theta)$ for $\rho=15$ and $10 \mathrm{~dB}$. Table I shows that the CRLB is approximately achieved for $\rho=20$ and $15 \mathrm{~dB}$, but not for $\rho=10 \mathrm{~dB}$. Based on this observation, we can describe the threshold phenomenon as follows. For sufficiently high SNRs (resp. relatively low SNRs), the maximum of the CCR falls in the vicinity of the maximum of the ACR (resp. spreads along the ACR) so the CRLB is (resp. is not) achieved.

\footnotetext{
${ }^{4}$ The ultra wideband (UWB) spectrum authorized for unlicensed use by the US federal commission of communications in May 2002 [48].

${ }^{5}$ We chose UWB pulses because they can achieve the CRLB at relatively low SNRs thanks to their relatively high fractional bandwidth (bandwidth to central
} frequency ratio). 
Consider now the pulse with $f_{c}=4 \mathrm{GHz}$. Fig. 2(b) and Table I show that for $\rho=20 \mathrm{~dB}$ all the samples of $M$ fall around the global maximum of $R(\theta-\Theta)$ and the CRLB is achieved, whereas for $\rho=15$ and $10 \mathrm{~dB}$ they spread along the local maxima of $R(\theta-\Theta)$ and the achieved MSE is much larger than the CRLB. Based on this observation, we can describe the ambiguity phenomenon as follows. For sufficiently high SNRs (resp. relatively low SNRs) the noise component $w(t)$ in the CCR $X_{s, r}(\theta)$ in (20) is not (resp. is) sufficiently high to fill the gap between the global maximum and the local maxima of the ACR. Consequently, for sufficiently high SNRs (resp. relatively low SNRs) the maximum of the CCR always falls around the global maximum (resp. spreads along the local maxima) of the ACR so the CRLB is (resp. is not) achieved. Obviously, the ambiguity phenomenon affects the threshold phenomenon because the SNR required to achieve the CRLB depends on the gap between the global and the local maxima.

We examine now the RMSE achieved at $\rho=20 \mathrm{~dB}$ for $f_{c}=4$ and $8 \mathrm{GHz}$; it is 3.5 times smaller with $f_{c}=4 \mathrm{GHz}$ than with $f_{c}=8 \mathrm{GHz}$ whereas the CRLB SQRT is 2 times smaller with the latter. In fact, the samples of $M$ do not fall all around the global maximum for $f_{c}=8 \mathrm{GHz}$. This amazing result (observed in [49] from experimental results) exhibits the significant loss in terms of accuracy if the CRLB is not achieved due to ambiguity. It also shows the necessity to design our system such that the CRLB be attained.

Let us finally note that due to the spread along the ACR, the MLE is only unbiased asymptotically (except for an even ACR with $\Theta$ located at the middle of $D_{\Theta}$ ). For the sake of conciseness, we have not shown numerical results regarding the mean.

\section{MIE-BAsed MLE Statistics ApPROXimation}

We have seen in Section III that the threshold phenomenon is due to the spreading of the estimates along the ACR. To characterize this phenomenon we split the a priori domain $D_{\Theta}$ into $N$ intervals $D_{n}=\left[d_{n}, d_{n+1}\right),\left(n=n_{1}, \ldots, n_{N}\right),\left(n_{1} \leq 0\right.$, $\left.n_{N} \geq 0\right)$ and write the PDF, mean and MSE of $\hat{\Theta}$ as

$$
\begin{aligned}
p(\theta) & =\sum_{n=n_{1}}^{n_{N}} P_{n} p_{n}(\theta) \\
\mu & =\int_{\Theta_{1}}^{\Theta_{2}} \theta p(\theta) d \theta=\sum_{n=n_{1}}^{n_{N}} P_{n} \mu_{n} \\
e & =\int_{\Theta_{1}}^{\Theta_{2}}(\theta-\Theta)^{2} p(\theta) d \theta=\sum_{n=n_{1}}^{n_{N}} P_{n}\left[\left(\Theta-\mu_{n}\right)^{2}+\sigma_{n}^{2}\right]
\end{aligned}
$$

where

$$
\begin{aligned}
P_{n} & =\mathbb{P}\left\{\hat{\Theta} \in D_{n}\right\} \\
& =\mathbb{P}\left\{\exists \xi \in D_{n}: X_{s, r}(\xi)>X_{s, r}(\theta), \forall \theta \in \cup_{n^{\prime} \neq n} D_{n^{\prime}}\right\}
\end{aligned}
$$

denotes the interval probability (i.e., probability that $\hat{\Theta}$ falls in $\left.D_{n}\right)$, and $p_{n}(\theta), \mu_{n}=\mathbb{E}\left\{\hat{\Theta}_{n}\right\}$ and $\sigma_{n}^{2}=\mathbb{E}\left\{\left(\hat{\Theta}_{n}-\mu_{n}\right)^{2}\right\}$ represent, respectively, the PDF, mean and variance of the interval $\operatorname{MLE}\left(\hat{\Theta}\right.$ given $\left.\hat{\Theta} \in D_{n}\right)$

$$
\hat{\Theta}_{n}=\hat{\Theta} \mid \hat{\Theta} \in D_{n} .
$$

The approximation of the mean is investigated because, as already mentioned, the MLE is only unbiased asymptotically. Denote by $\theta_{n}$ a testpoint selected in $D_{n}$ and let $X_{n}=X_{s, r}\left(\theta_{n}\right)=$ $\alpha R_{n}+w_{n}$ with $R_{n}=R_{s}\left(\theta_{n}, \Theta\right)$ and $w_{n}=w\left(\theta_{n}\right)$. Using (3), $P_{n}$ in (23) can be approximated by

$$
\begin{aligned}
\tilde{P}_{n}= & \mathbb{P}\left\{X_{n}>X_{n^{\prime}}, \forall n^{\prime} \neq n\right\}=\int_{-\infty}^{+\infty} d x_{n} \int_{-\infty}^{x_{n}} d x_{n_{1}} \\
& \ldots \int_{-\infty}^{x_{n}} d x_{n-1} \int_{-\infty}^{x_{n}} d x_{n+1} \cdots \int_{-\infty}^{x_{n}} p_{X}(x) d x_{n_{N}}
\end{aligned}
$$

where

$$
p_{X}(x)=\frac{1}{(2 \pi)^{\frac{N}{2}}\left|C_{X}\right|^{\frac{1}{2}}} e^{-\frac{\left(x-\mu_{X}\right) C_{X}^{-1}\left(x-\mu_{X}\right)^{T}}{2}}
$$

represents the PDF of $X=\left(X_{n_{1}} \cdots X_{n_{N}}\right)^{T}$ with $\mu_{X}=\left(\mu_{X_{n_{1}}} \cdots \mu_{X_{n_{N}}}\right)^{T}=\alpha\left(R_{n_{1}} \cdots R_{n_{N}}\right)^{T}$ being its mean and $C_{X}=\frac{N_{0}}{2}\left[R_{s}\left(\theta_{n}, \theta_{n^{\prime}}\right)\right]_{n, n^{\prime}=n_{1}, \ldots, n_{N}}$ its covariance matrix.

The accuracy of the approximation in (25) depends on the choice of the intervals and the testpoints. For an oscillating ACR we consider an interval around each local maximum and choose the abscissa of the local maximum as a testpoint, whereas for a non-oscillating ACR we split $D_{\Theta}$ into equal intervals and choose the center $\theta_{n}=\frac{d_{n}+d_{n+1}}{2}$ of each interval as a testpoint. For both oscillating and non-oscillating ACRs, $D_{0}$ contains the global maximum and $\theta_{0}$ is equal to $\Theta$.

The testpoints are chosen as the roots of the ACR (except for $\theta_{0}=\Theta$ ) in [18], [36], [37], [40]-[42], as the local extrema abscissa in [1], and as the local maxima abscissa in [15], [41], [43], [44].

\section{A. Computation of the Interval Probability}

We consider here the computation of the approximate interval probability $\tilde{P}_{n}$ in (25).

1) Numerical Approximation: To the best of our knowledge there is no closed form expression for the integral in (25) for correlated $X_{n}$. However, it can be computed numerically using for example the MATLAB function QSCMVNV (written by Genz based on [50]-[53]) that computes the multivariate normal probability with integration region specified by a set of linear inequalities in the form $b_{1}<B\left(X-\mu_{X}\right)<b_{2}$. Using QSCMVNV, $\tilde{P}_{n}$ can be approximated by:

$$
P_{n}^{(1)}=\operatorname{QSCMVNV}\left(N_{p}, C_{X}, b_{1}, B, b_{2}\right)
$$

where $N_{p}$ is the number of points used by the algorithm (e.g., $\left.N_{p}=3000\right), b_{1}=(-\infty \cdots-\infty)^{T}$ and $b_{2}=$ $\mu_{X_{n}}-\left(\mu_{X_{n_{1}}} \cdots \mu_{X_{X_{-1}}} \mu_{X_{n+1}} \cdots \mu_{X_{n_{N}}}\right)^{T}$ two $(N-1)$-column

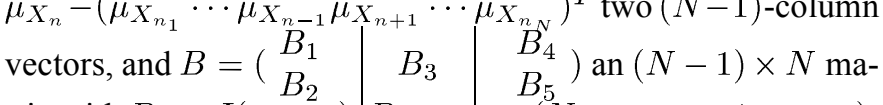
trix with $B_{1}=I\left(n-n_{1}\right), B_{2}=\operatorname{zeros}\left(N+n_{1}-n-1, n-n_{1}\right)$, $B_{3}=-\operatorname{ones}(N-1,1), B_{4}=\operatorname{zeros}\left(N-n_{N}+n-1, n_{N}-n\right)$ and $B_{5}=I\left(n_{N}-n\right)^{6}$.

${ }^{6}$ We denote by $I(k)$ the identity matrix of rank $k$, and zeros $\left(k_{1}, k_{2}\right)$ and ones $\left(k_{1}, k_{2}\right)$ the zero and one matrices of dimension $k_{1} \times k_{2}$. 


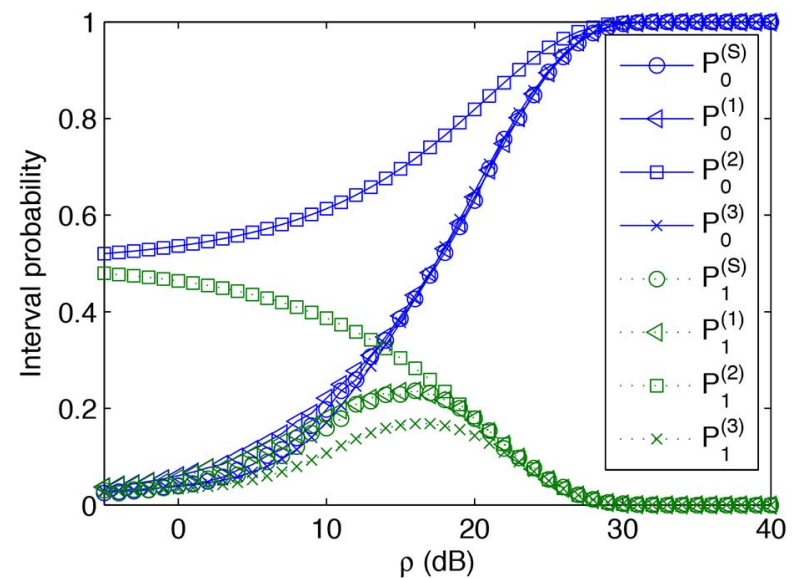

Fig. 3. Simulated interval probability $P_{n}^{(S)}$, the approximations $P_{n}^{(1)}$ and $P_{n}^{(3)}$, and the AUB $P_{n}^{(2)}$ for $n=0,1$ w.r.t. the SNR.

2) Analytic Approximation: Denote by $Q(y)=$ $\frac{1}{\sqrt{2 \pi}} \int_{y}^{\infty} e^{-\frac{\xi^{2}}{2}} d \xi$ the Q function. As $\mathbb{P}\left\{A_{1} \cap A_{2}\right\} \leq \mathbb{P}\left\{A_{1}\right\}$, we can upper bound $\tilde{P}_{n}$ in (25) by:

$$
P_{n}^{(2)}= \begin{cases}P\left(\theta_{0}, \theta_{1}\right) & n=0 \\ P\left(\theta_{n}, \theta_{0}\right) & n \neq 0\end{cases}
$$

where

$$
\begin{aligned}
P\left(\theta, \theta^{\prime}\right) & =\mathbb{P}\left\{X_{s, r}(\theta)>X_{s, r}\left(\theta^{\prime}\right)\right\} \\
& =Q\left(\sqrt{\frac{\rho}{2}} \frac{R\left(\theta^{\prime}, \Theta\right)-R(\theta, \Theta)}{\sqrt{1-R\left(\theta, \theta^{\prime}\right)}}\right)
\end{aligned}
$$

with $R(\theta, \Theta)=\frac{R_{s}(\theta, \Theta)}{E}$ denoting the normalized ACR. $P\left(\theta, \theta^{\prime}\right)$ is obtained (28) from (3) and (6) by noticing that $X_{s, r}(\theta)-X_{s, r}\left(\theta^{\prime}\right) \sim \mathcal{N}\left(\alpha\left[R_{s}(\theta, \Theta)-R_{s}\left(\theta^{\prime}, \Theta\right)\right], N_{0}\left[E_{s}-\right.\right.$ $\left.\left.R_{s}\left(\theta, \theta^{\prime}\right)\right]\right)^{7}$. If $N$ approaches infinity, then both $\sum_{n=n_{1}}^{n_{N}} P_{n}^{(2)}$ and the MSEA in (22) will approach infinity.

Using (27), we propose the following approximation:

$$
P_{n}^{(3)}=\frac{P_{n}^{(2)}}{\sum_{n=n_{1}}^{n_{N}} P_{n}^{(2)}} .
$$

In this subsection we have seen that the interval probability $P_{n}$ in (23) can be approximated by $P_{n}^{(1)}$ in (26) or $P_{n}^{(3)}$ in (29), and upper bounded by $P_{n}^{(2)}$ in (27).

The UB $P_{n}^{(2)}$ is adopted in [1], [15], [41], [43], [44] with minor modifications; in fact, $\tilde{P}_{0}$ is approximated by one in [1] and by $1-\sum_{n \neq 0} P_{n}^{(2)}$ in [15], [41], [43], [44]. In the special case where $X_{n_{1}}, \ldots, X_{-1}, X_{1}, \ldots, X_{n_{N}}$ are independent and identically distributed such as in [18], [36], [37], [40]-[42] thanks to the cardinal sine ACR, then $\tilde{P}_{n}=\frac{\tilde{P}_{A}}{N-1}, \forall n \neq 0$, and $\tilde{P}_{0}=1-\tilde{P}_{A}\left(\tilde{P}_{A}\right.$ is the approximate probability of ambiguity); consequently, the MSEA in (22) can be written as the sum of two terms: $e \approx \tilde{P}_{A} e_{U}+\tilde{P}_{0} c(\Theta) ; \tilde{P}_{0}$ can be calculated by performing one-dimensional integration. If $X_{0} \sim \mathcal{N}\left(\alpha E_{s}, \frac{N_{0}}{2} E_{s}\right)$ and $X_{n} \sim \mathcal{N}\left(0, \frac{N_{0}}{2} E_{s}\right), \forall n \neq 0$, like in [18], [36], [37], [41] then $P_{A}$ can be upper bounded using the union bound [36].

As an example, to evaluate the accurateness of $P_{n}^{(1)}$ in (26) and $P_{n}^{(3)}$ in (29) and to compare them to $P_{n}^{(2)}$ in (27), we consider the pulse in (21) with $f_{c}=6.85 \mathrm{GHz}, T_{w}=2 \mathrm{~ns}, \Theta=0$

\footnotetext{
${ }^{7} \mathcal{N}(m, v)$ stands for the normal distribution of mean $m$ and variance $v$
}

and $D_{\Theta}=[-2,1.5] T_{w}$. In Fig. 3 we show for $n=0$ and 1 , the interval probability $P_{n}^{(S)}$ obtained by simulation based on 10000 trials, $P_{n}^{(1)}, P_{n}^{(2)}$ and $P_{n}^{(3)}$, all versus the SNR. We can see that $P_{n}^{(S)}$ converges to $\frac{1}{N}$ at low SNRs for all intervals; however, it converges to 1 at high $\operatorname{SNRs}\left(P_{0}^{S}=0.99\right.$ for $\left.\rho \approx 30 \mathrm{~dB}\right)$ for $n=0$ (probability of non-ambiguity) and to 0 for $n \neq 0$. Both $P_{n}^{(1)}$ and $P_{n}^{(3)}$ are very accurate and closely follow $P_{n}^{(S)}$. The UB $P_{n}^{(2)}$ is not tight at low SNRs; it converges to $0.5 \forall n$ instead of $\frac{1}{N}$ due to (28). However, it converges to 1 (resp. 0) for $n=0$ (resp. $n \neq 0$ ) at high SNRs simultaneously with $P_{n}^{(S)}$ so it can be used to determine accurately the asymptotic region.

\section{B. Statistics of the Interval MLE}

We approximate here the statistics of the interval MLE $\hat{\Theta}_{n}$ in (24). We have already mentioned in Section IV that for an oscillating (resp. a non-oscillating) ACR we consider an interval around each local maximum (resp. split the a priori domain into equal intervals); the global maximum is always contained in $D_{0}$. Accordingly, the ACR inside a given interval is either increasing then decreasing or monotone (i.e., increasing, decreasing or constant).

As the distribution of $\hat{\Theta}_{n}$ should follow the shape of the ACR in the considered interval, the interval variance is upper bounded by the variance of uniform distribution in $D_{n}=\left[d_{n}, d_{n+1}\right]$. Therefore, the interval mean $\mu_{n}$ and variance $\sigma_{n}^{2}$ can be approximated by

$$
\begin{aligned}
\mu_{n, U} & =\frac{d_{n}+d_{n+1}}{2} \\
\sigma_{n, U}^{2} & =\frac{\left(d_{n+1}-d_{n}\right)^{2}}{12} .
\end{aligned}
$$

For intervals with local minima (not considered here), the ACR decreases then increases so $\sigma_{n}^{2}$ is upper bounded by the variance of a Bernoulli distribution of two equiprobable atoms:

$$
\sigma_{n, \max }^{2}=\frac{\left(d_{n+1}-d_{n}\right)^{2}}{4}>\sigma_{n, U}^{2}
$$

In [1], it is assumed that $\sigma_{n}^{2}$ is upper bounded by $\sigma_{i, U}^{2}$ in (31) even for intervals with local minima. See [54], [55] for further information on the maximum variance.

The CCR $X_{s, r}(\theta)$ in (3) can be approximated inside $D_{n}$ by its Taylor series expansion about $\theta_{n}$ limited to second order:

$$
\begin{aligned}
X_{s, r}(\theta)= & \alpha R_{s}(\theta, \Theta)+w(\theta) \\
\approx & \left(\alpha R_{n}+w_{n}\right)+\left(\alpha \dot{R}_{n}+\dot{w}_{n}\right)\left(\theta-\theta_{n}\right) \\
& +\left(\alpha \ddot{R}_{n}+\ddot{w}_{n}\right) \frac{\left(\theta-\theta_{n}\right)^{2}}{2}
\end{aligned}
$$

where $\dot{w}_{n}=\dot{w}\left(\theta_{n}\right), \ddot{w}_{n}=\ddot{w}\left(\theta_{n}\right), \dot{R}_{n}=\dot{R}_{s}\left(\theta_{n}, \Theta\right)$ and $\ddot{R}_{n}=$ $\ddot{R}_{s}\left(\theta_{n}, \Theta\right)$. Let $\nu_{n}$ be the correlation coefficient of $\dot{w}_{n}$ and $\ddot{w}_{n}$. Then, from (5), we can show that

$$
\begin{gathered}
\dot{w}_{n} \sim \mathcal{N}\left(0, \sigma_{\dot{w}_{n}}^{2}\right) \\
\ddot{w}_{n} \sim \mathcal{N}\left(0, \sigma_{\ddot{w}_{n}}^{2}\right)
\end{gathered}
$$

with

$$
\begin{aligned}
& \sigma_{\dot{w}_{n}}^{2}=\frac{N_{0}}{2} \int_{-\infty}^{+\infty} \dot{s}^{2}\left(t ; \theta_{n}\right) d t=\frac{N_{0}}{2} E_{\dot{s}}\left(\theta_{n}\right) \\
& \sigma_{\ddot{w}_{n}}^{2}=\frac{N_{0}}{2} \int_{-\infty}^{+\infty} \ddot{s}^{2}\left(t ; \theta_{n}\right) d t=\frac{N_{0}}{2} E_{\ddot{s}}\left(\theta_{n}\right)
\end{aligned}
$$




$$
\nu_{n}=\frac{\mathbb{E}\left\{\dot{w}_{n} \ddot{w}_{n}\right\}}{\sigma_{\dot{w}_{n}} \sigma_{\ddot{w}_{n}}}=\frac{\int_{-\infty}^{+\infty} \dot{s}\left(t ; \theta_{n}\right) \ddot{s}\left(t ; \theta_{n}\right) d t}{\sqrt{E_{\dot{s}}\left(\theta_{n}\right) E_{\ddot{s}}\left(\theta_{n}\right)}} .
$$

Let us first consider an interval with monotone ACR. By neglecting $\ddot{w}_{n}$ and $\ddot{R}_{n}$ in (33) (linear approximation), we can approximate the interval MLE by:

$$
\begin{aligned}
\hat{\Theta}_{n} & =\underset{\theta \in D_{n}}{\operatorname{argmax}}\left\{X_{s, r}(\theta)\right\} \\
& \approx \begin{cases}d_{n} & \alpha \dot{R}_{n}+\dot{w}_{n}<0 \\
d_{n+1} & \alpha \dot{R}_{n}+\dot{w}_{n}>0 \\
\frac{d_{n, 1}+d_{n, 2}}{2} & \alpha \dot{R}_{n}+\dot{w}_{n}=0 .\end{cases}
\end{aligned}
$$

As $\mathbb{P}\left\{\alpha \dot{R}_{n}+\dot{w}_{n}=0\right\}=0$, the latter approximation follows a two atoms Bernoulli distribution with probability, mean and variance given from (9), (34) and (36) by:

$$
\begin{aligned}
\mathbb{P}\left\{d_{n}\right\} & =1-\mathbb{P}\left\{d_{n+1}\right\}=\mathbb{P}\left\{-\dot{w}_{n}>\alpha \dot{R}_{n}\right\} \\
& =Q\left(\frac{\alpha \dot{R}_{n}}{\sigma_{\dot{w}_{n}}}\right)=Q\left(\sqrt{\frac{\rho \dot{R}_{n}^{2}}{E_{s} E_{\dot{s}}\left(\theta_{n}\right)}}\right) \\
\mu_{n, B} & =d_{n} \mathbb{P}\left\{d_{n}\right\}+d_{n+1} \mathbb{P}\left\{d_{n+1}\right\} \\
\sigma_{n, B}^{2} & =\mathbb{P}\left\{d_{n}\right\} \mathbb{P}\left\{d_{n+1}\right\}\left(d_{n+1}-d_{n}\right)^{2}
\end{aligned}
$$

where $\sigma_{n, B}^{2}$ is upper bounded by $\sigma_{n, \max }^{2}$ in (32) and reaches it for $\mathbb{P}\left\{d_{n}\right\}=0.5 ; \mathbb{P}\left\{d_{n}\right\}=0.5$ just means that $\hat{\Theta}_{n}$ is uniformly distributed in $D_{n}$ (because $\hat{\Theta}_{n}$ can fall anywhere inside $D_{n}$ ); therefore, $\mu_{n}$ and $\sigma_{n}^{2}$ can be approximated by:

$$
\begin{aligned}
\mu_{n, 1, c} & =\mu_{n, B} \\
\sigma_{n, 1, c}^{2} & =\min \left\{\sigma_{n, U}^{2}, \sigma_{n, B}^{2}\right\} .
\end{aligned}
$$

By neglecting $\dot{w}_{n}$ in (33) and (39) (because $\sigma_{n}^{2}<<\left(\Theta-\mu_{n}\right)^{2}$ for $n \neq 0$, see (22)) we obtain the following approximation:

$$
\begin{aligned}
& \mu_{n, 2, c}= \begin{cases}d_{n} & \dot{R}_{n}<0 \\
d_{n+1} & \dot{R}_{n}>0 \\
\frac{d_{n}+d_{n+1}}{2} & \dot{R}_{n}=0\end{cases} \\
& \sigma_{n, 2, c}^{2}=0 .
\end{aligned}
$$

Consider now an interval with a local maximum. By neglecting $\ddot{w}_{n}$ in (33), and taking into account that $\dot{R}_{n}=0$ (local maximum), $\hat{\Theta}_{n}$ can be approximated by:

$$
\hat{\Theta}_{n}=\underset{\theta \in D_{n}}{\operatorname{argmax}}\left\{X_{s, r}(\theta)\right\} \approx \theta_{n}-\frac{\dot{w}_{n}}{\alpha \ddot{R}_{n}}
$$

which follows a normal distribution whose PDF, mean and variance can be obtained from (8), (34), (36) and (45):

$$
\begin{aligned}
p_{n, N}(\theta) & =\frac{1}{\sqrt{2 \pi} \sigma_{n, N}} e^{-\frac{\left(\theta-\mu_{n, N}\right)^{2}}{2 \sigma_{n, N}^{2}}} \\
\mu_{n, N} & =\theta_{n} \\
\sigma_{n, N}^{2} & =\frac{\sigma_{\dot{w}_{n}}^{2}}{\alpha^{2} \ddot{R}_{n}^{2}}=\frac{\frac{N_{0}}{2} E_{\dot{s}}\left(\theta_{n}\right)}{\alpha^{2} \ddot{R}_{n}^{2}}=c \frac{-\ddot{R}_{0} E_{\dot{s}}\left(\theta_{n}\right)}{\ddot{R}_{n}^{2}} .
\end{aligned}
$$

For $n=0, \sigma_{n, N}^{2}$ is equal to the CRLB in (8) since $-\ddot{R}_{0}=$ $E_{\dot{s}}\left(\theta_{0}\right)$. To take into account that $D_{n}$ is finite, we propose from (46), (47) and (48) the following approximation:

$$
\begin{aligned}
\mu_{n, 1, o} & =\int_{d_{n}}^{d_{n+1}} \theta p_{n, 1, o}(\theta) d \theta \approx \theta_{n} \\
\sigma_{n, 1, o}^{2} & =\int_{d_{n}}^{d_{n+1}}\left(\theta-\mu_{n, 1, o}\right)^{2} p_{n, 1, o}(\theta) d \theta \\
& \approx \min \left\{\sigma_{n, N}^{2}, \sigma_{n, U}^{2}\right\}
\end{aligned}
$$

where $p_{n, 1, o}(\theta)=\frac{p_{n, N}(\theta)}{\int_{d_{n}}^{d_{n}+1} p_{n, N}(\theta) d \theta}$. By neglecting $w(\theta)$ in (33) and (45), we obtain the following approximation:

$$
\begin{aligned}
& \mu_{n, 2, o}=\theta_{n} \\
& \sigma_{n, 2, o}^{2}=0 .
\end{aligned}
$$

For both oscillating and non-oscillating ACRs, $D_{0}$ contains the global maximum. To guarantee the convergence of the MSEA in (22) to the CRLB, $\mu_{0}$ and $\sigma_{0}^{2}$ should always be approximated using (49) and (50) by:

$$
\begin{aligned}
& \mu_{0,0}=\Theta \\
& \sigma_{0,0}^{2}=\min \left\{c, \sigma_{0, U}^{2}\right\} .
\end{aligned}
$$

For TOA estimation, we can write (40) and (48) as $\mathbb{P}\left\{d_{n}\right\}=$ $Q\left(\sqrt{\rho} \frac{\dot{R}_{n}}{E_{s} \beta_{s}}\right)$ and $\sigma_{n, N}^{2}=c \frac{\ddot{R}_{0}^{2}}{\ddot{R}_{n}^{2}}$.

We have seen in this subsection that the interval mean and variance can be approximated by

- $\mu_{0,0}$ in (53) and $\sigma_{0,0}^{2}$ in (54) for $n=0$.

- $\mu_{n, U}$ in (30) and $\sigma_{n, U}^{2}$ in (31), $\mu_{n, 1, c}$ in (41) and $\sigma_{n, 1, c}^{2}$ in (42), or $\mu_{n, 2, c}$ in (43) and $\sigma_{n, 2, c}^{2}$ in (44) for intervals with monotone ACR.

- $\mu_{n, U}$ and $\sigma_{n, U}^{2}, \mu_{n, 1, o}$ in (49) and $\sigma_{n, 1, o}^{2}$ in (50), or $\mu_{n, 2, o}$ in (51) and $\sigma_{n, 2, o}^{2}$ in (52) for intervals with local maxima. In [18], [36], [37], [40], [42] (resp. [15], [41], [43], [44]) $\sigma_{n}^{2}$ is approximated by $\sigma_{n, U}^{2}$ (resp. $\left.\sigma_{n, 2, o}^{2}\right)$. They all approximate $\mu_{n}$ by $\theta_{n}$ and $\sigma_{0}^{2}$ by the asymptotic MSE (equal to the CRLB if the considered estimator is asymptotically efficient).

To evaluate the accurateness of $\sigma_{n, U}^{2}$ in (31) and $\sigma_{n, 1, o}^{2}$ in (50), we consider the pulse in (21) with $f_{c}=8 \mathrm{GHz}, T_{w}=$ $0.6 \mathrm{~ns}, D_{\Theta}=[-1.5,1.5] T_{w}$ and $\rho=10 \mathrm{~dB}$. In Fig. 4 we show the approximate interval standard deviations (STD) $\sigma_{n, U}$ and $\sigma_{n, 1, o}$, and the STD $\sigma_{n, S}$ obtained by simulation based on 50000 trials, w.r.t. the interval number $n=-6, \ldots, 6$. We can see that $\sigma_{n, S}$ is upper bounded by $\sigma_{n, U}$ as expected and that $\sigma_{n, 1, o}$ follows $\sigma_{n, S}$ closely. The smallest variance corresponds to $n=0$ because the curvature of $R_{s}(\theta, \Theta)$ reaches its maximum at $\theta=\Theta$.

Before ending this section, we would like to highlight our contributions regarding the MIE. We have proposed two approximations for the interval probability when $X_{n_{1}}, \ldots, X_{n_{N}}$ are correlated. We have shown in Fig. 3 how our approximations are accurate. To the best of our knowledge all previous authors adopt the McAulay probability UB (except for the case where $X_{n_{1}}, \ldots, X_{n_{N}}$ are independent thanks to the cardinal sine ACR). We have proposed two new approximations for the interval mean and variance, one for intervals with monotone 


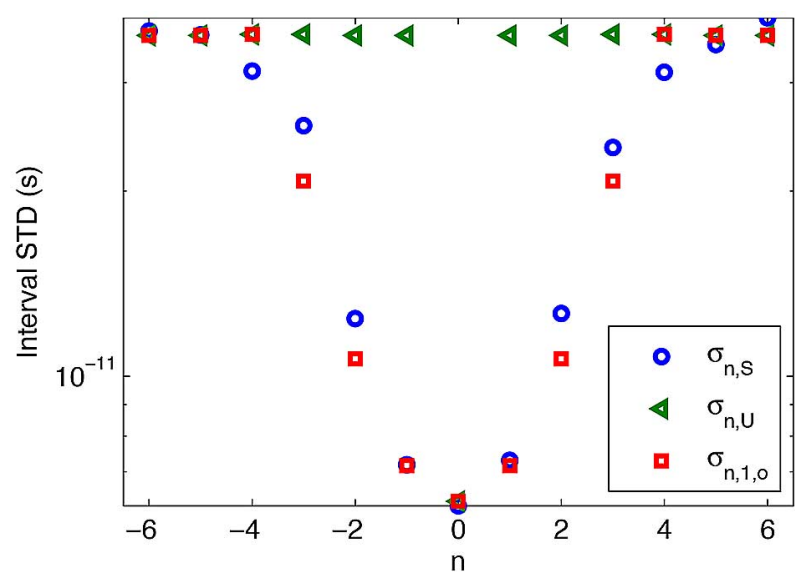

Fig. 4. Simulated interval STD $\sigma_{n, S}$ and approximations $\sigma_{n, U}$ and $\sigma_{n, 1, o}$ w.r.t. the interval number $n=-6, \ldots, 6$ for $\rho=10 \mathrm{~dB}$.

ACRs and one for intervals with local maxima. We have seen in Fig. 4 how our approximations are accurate. To the best of our knowledge all previous authors either upper bound the interval variance or neglect it. Thanks to the proposed probability approximations our MSEAs (e.g., $e_{1,1, c}$ in Fig. 6) are highly accurate and outperform the MSE UB of McAulay ( $e_{2, U}$ in Fig. 7) and thanks to the proposed interval variance approximations the MSEA is improved ( $e_{1, U}$ and $e_{1,2, c}$ outperform $e_{1,1, c}$ in Fig. 6). We have applied the MIE to non-oscillating ACRs. To the best of our knowledge this case is not considered before.

\section{AN AUB AND AN MSEA BASED ON THE INTERVAL PROBABILITY}

In this section we propose an AUB (Section V.A) and an MSEA (Section V.B), both based on the interval probability approximation $P_{n}^{(3)}$ in (29).

\section{A. An $A U B$}

As $P_{n}^{(3)}$ approximates the probability that $\hat{\Theta}$ falls in $D_{n}$, the PDF of $\hat{\Theta}$ can be approximated by the limit of $P_{n}^{(3)}$ as $N$ (number of intervals) approaches infinity (so that the width of $D_{n}$ approaches zero). Accordingly we can write the approximate PDF, mean and MSE of $\hat{\Theta}$ as

$$
\begin{aligned}
p_{M}(\theta) & =\lim _{N \rightarrow \infty} P_{n}^{(3)}=\frac{P(\theta, \Theta)}{\int_{\Theta_{1}}^{\Theta_{2}} P(\theta, \Theta) d \theta} \\
\mu_{M} & =\int_{\Theta_{1}}^{\Theta_{2}} \theta p_{M}(\theta) d \theta \\
e_{M} & =\int_{\Theta_{1}}^{\Theta_{2}}(\theta-\Theta)^{2} p_{M}(\theta) d \theta .
\end{aligned}
$$

We will see in Section VII that $e_{M}$ acts as an UB and also converges to a multiple of the CRLB. In fact, $p_{M}(\theta)$ overestimates the true PDF of $\hat{\Theta}$ in the vicinity of $\Theta$ because it is obtained from $P_{n}^{(3)}$ which is in turn obtained from the interval probability UB $P_{n}^{(2)}$ in (27).

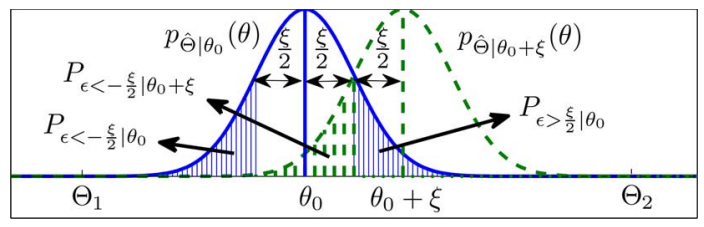

Fig. 5. Decision problem with two equiprobable hypotheses: $H_{1}: \Theta=\theta_{0}$ and $H_{2}: \Theta=\theta_{0}+\xi$.

\section{B. An MSEA}

To guarantee the convergence of the MSEA to the CRLB, we approximate the PDF of $\hat{\Theta}$ inside $D_{0} \approx\left[\Theta-\frac{\theta_{1}-\Theta}{2}, \Theta+\frac{\theta_{1}-\Theta}{2}\right.$ ) by $p_{0, N}(\theta)$ in (46) $(\Theta$ is the mean and $c(\Theta)$ is the MSE) and outside $D_{0}$ by $p_{M}^{\prime}(\theta)=P(\theta, \Theta) / \int_{D_{\Theta} \backslash D_{0}} P(\theta, \Theta) d \theta$ (the corresponding mean and MSE are $\mu_{M}^{\prime}=\int_{D_{\Theta} \backslash D_{0}} \theta p_{M}^{\prime}(\theta) d \theta$ and $\left.e_{M}^{\prime}=\int_{D_{\Theta} \backslash D_{0}}(\theta-\Theta)^{2} p_{M}^{\prime}(\theta) d \theta\right)$, and propose the following approximation:

$$
\begin{aligned}
p_{M N}(\theta) & =\left(1-\tilde{P}_{A}\right) p_{0, N}(\theta)+\tilde{P}_{A} p_{M}^{\prime}(\theta) \\
\mu_{M N} & =\left(1-\tilde{P}_{A}\right) \Theta+\tilde{P}_{A} \mu_{M}^{\prime} \\
e_{M N} & =\left(1-\tilde{P}_{A}\right) c(\Theta)+\tilde{P}_{A} e_{M}^{\prime}
\end{aligned}
$$

where $\tilde{P}_{A}=2 P\left(\theta_{1}, \Theta\right)$ approximates the probability that $\hat{\Theta}$ falls outside $D_{0}$. With oscillating ACRs, $\theta_{1}$ is the abscissa of the first local maximum after the global one; thus, $\theta_{1} \approx \Theta+\frac{1}{f_{c}(\Theta)}$. With non-oscillating ACRs, the vicinity of the maximum is not clearly marked off; so, we empirically take $\theta_{1}=\Theta+\frac{\pi}{4 \beta_{s}(\Theta)}$.

The first contribution in this section is the AUB $e_{M}$ which is very tight (as will be seen in Figs. 7 and 9) and also very easy to compute. The second one is the highly accurate MSEA $e_{M N}$ (as will be seen in Figs. 6 and 8); to the best of our knowledge, this is the first approximation expressed as the sum of two terms when $X_{n_{1}}, \ldots, X_{n_{N}}$ are correlated (see [1], [15], [41], [43], [44]).

\section{ALBS}

In this section we derive an ALB based on the Taylor series expansion of the noise limited to second order (Section VI.A) and a family of ALBs by employing the principle of binary detection which is first used by Ziv and Zakai [2] to derive LBs for Bayesian parameters (Section VI.B).

\section{A. An ALB Based on the Second Order Taylor Series Expansion of Noise}

From (33), the MLE of $\Theta$ can be approximated by:

$$
\hat{\Theta}=\underset{\theta}{\operatorname{argmax}}\left\{X_{s, r}(\theta)\right\} \approx \hat{\Theta}_{C}=\Theta-\frac{\dot{w}_{0}}{\alpha \ddot{R}_{0}+\ddot{w}_{0}}
$$

where $\dot{w}_{0} /\left(\alpha \ddot{R}_{0}+\ddot{w}_{0}\right)$ is a ratio of two normal variables. Statistics of normal variable ratios are studied in [56]-[58].

Let $\operatorname{sign}(\xi)=1$ (resp. -1$)$ for $\xi \geq 0($ resp. $\xi<0), \delta^{4}(\theta)=$ $E_{\ddot{s}}(\theta) / E_{s}, h=\operatorname{sign}\left(\nu_{0}\right) \sigma_{\dot{w}_{0}} \sqrt{1-\nu_{0}^{2}}, a_{1}=\nu_{0} \sigma_{\dot{w}_{0}} / \sigma_{\ddot{w}_{0}}$, $a_{2}=\sigma_{\ddot{w}_{0}} / h, a_{3}=\alpha \ddot{R}_{0} a_{1} / h, a_{4}=-\alpha \ddot{R}_{0} / \sigma_{\ddot{w}_{0}}=$ $\sqrt{\rho} \beta^{2}(\Theta) / \delta^{2}(\Theta), q(\xi)=\left(a_{3} \xi+a_{4}\right) / \sqrt{1+\xi^{2}}$. We can show from [57] that $\hat{\Theta}_{C}$ in (61) is distributed as:

$$
\hat{\Theta}_{C} \sim \Theta+a_{1}+\frac{\chi}{a_{2}}
$$




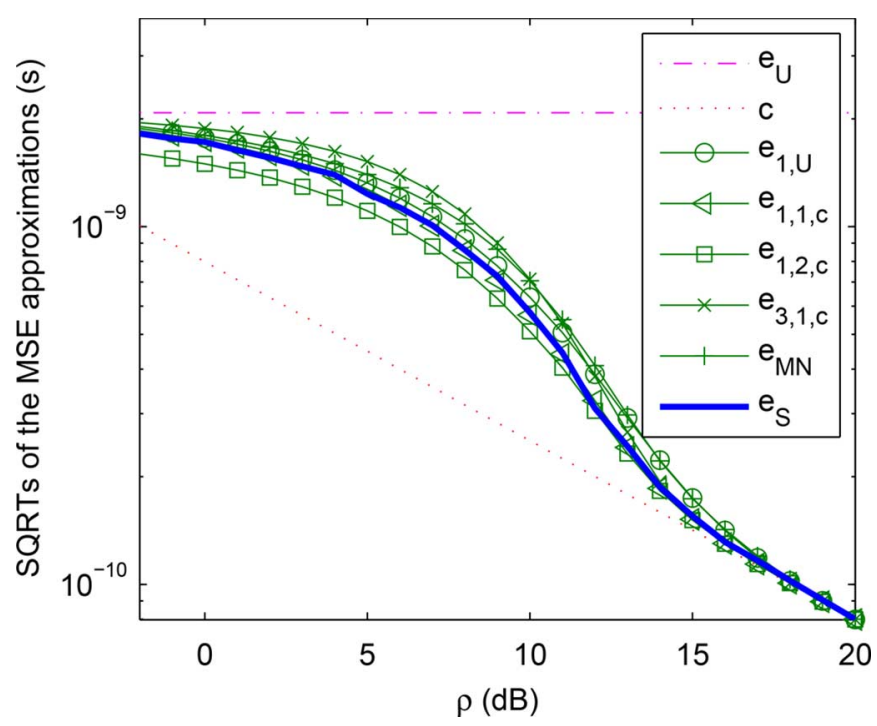

Fig. 6. Baseband: SQRTs of the max. MSE $e_{U}$, the CRLB $c$, the MSEAs $e_{1, U}$, $e_{1,1, c}, e_{1,2, c}, e_{3,1, c}$ and $e_{M N}$, and the simulated MSE $e_{S}$, w.r.t. the SNR

where the PDF of $\chi$ is given by:

$p_{\chi}(\xi)=\frac{e^{-\frac{a_{3}^{2}+a_{4}^{2}}{2}}}{\pi\left(1+\xi^{2}\right)}\left\{1+\sqrt{2 \pi} q(\xi) e^{\frac{q^{2}(\xi)}{2}}\left(\frac{1}{2}-Q[q(\xi)]\right)\right\}$.

From (63) we can approximate the PDF, mean, variance and MSE of $\hat{\Theta}_{C}$ by

$$
\begin{aligned}
p_{C}(\theta) & =\operatorname{sign}\left(\nu_{0}\right) a_{2} p_{\chi}\left[a_{2}\left(\theta-\Theta-a_{1}\right)\right] \\
\mu_{C} & =\int_{\Theta_{1}}^{\Theta_{2}} \theta p_{C}(\theta) d \theta \\
\sigma_{C}^{2} & =\int_{\Theta_{1}}^{\Theta_{2}}\left(\theta-\mu_{C}\right)^{2} p_{C}(\theta) d \theta \\
e_{C} & =\left(\mu_{C}-\Theta\right)^{2}+\sigma_{C}^{2} .
\end{aligned}
$$

Note that the moments $\int_{-\infty}^{\infty} \xi^{i} p_{\chi}(\xi) d \xi, i=1,2, \cdots$ (infinite domain) are infinite like with Cauchy distribution [57]. We will see in Section VII that $e_{C}$ behaves as an LB; this result can be expected from the approximation in (33) where the expansion of the noise is limited to second order.

\section{B. Binary Detection Based ALBs}

Let $\tilde{\Theta}$ be an estimator of $\Theta, \epsilon \mid \theta=\tilde{\Theta}-\Theta$ the estimation error given $\Theta=\theta, p_{|\epsilon| \mid \theta}(\xi)$ the PDF of $|\epsilon|$, and $P_{|\epsilon|>\xi \mid \theta}$ the probability that $|\epsilon|>\xi$. For $\Theta=\theta_{0}$, the MSE of $\Theta$ can be written as [59]:

$$
\begin{aligned}
e \mid \theta_{0}=\int_{0}^{\epsilon_{\max }} \xi^{2} p_{|\epsilon| \mid \theta_{0}}(\xi) d \xi & =2 \int_{0}^{\epsilon_{\max }} \xi P_{|\epsilon>\xi| \theta_{0}} d \xi \\
-\left.\left\{\xi^{2} P_{|\epsilon|>\xi^{\prime} \theta_{0}}\right\}\right|_{0} ^{\epsilon_{\max }}= & \frac{1}{2} \int_{0}^{2 \epsilon_{\max }} \xi P_{|\epsilon|>\frac{\xi}{2} \mid \theta_{0}} d \xi
\end{aligned}
$$

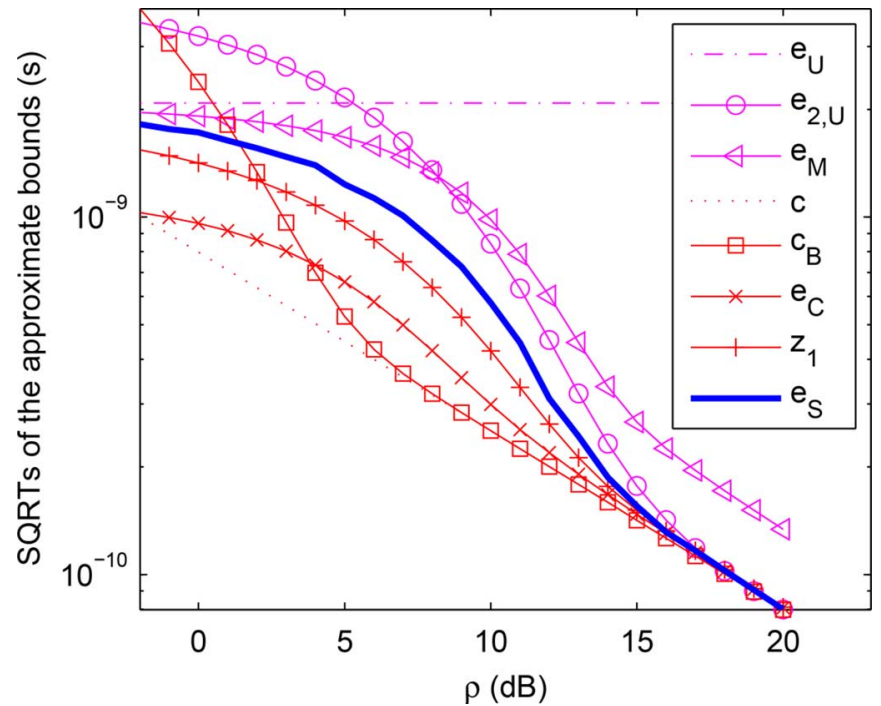

Fig. 7. Baseband: SQRTs of the max. $\operatorname{MSE} e_{U}$, the $\mathrm{AUBs} e_{2, U}$ and $e_{M}$, the $\operatorname{CRLB} c$, the $\operatorname{BLB} c_{B}$, the ALBs $e_{C}$ and $z_{1}$, and the simulated MSE $e_{S}$, w.r.t. the SNR.

where $\epsilon_{\max }=\max \left\{\Theta_{2}-\theta_{0}, \theta_{0}-\Theta_{1}\right\}$. By assuming $P_{\epsilon>\frac{\xi}{2} \mid \theta}$ and $P_{\epsilon<-\frac{\xi}{2} \mid \theta}$ constant $\forall \theta \in D_{\Theta}$, we can write 8 :

$$
\begin{aligned}
P_{|\epsilon|>\frac{\xi}{2} \mid \theta_{0}} & =2\left[\frac{1}{2} P_{\epsilon>\frac{\xi}{2} \mid \theta_{0}}+\frac{1}{2} P_{\epsilon<-\frac{\xi}{2} \mid \theta_{0}}\right] \\
& \approx 2\left\{\begin{array}{l}
P_{\epsilon_{1}}=\frac{1}{2} P_{\epsilon>\frac{\xi}{2}} \mid \theta_{0}-\xi \\
P_{\epsilon_{2}}=\frac{1}{2} P_{\epsilon>\frac{\xi}{2}} \mid \theta_{0}+\frac{1}{2} P_{\epsilon<-\frac{\xi}{2} \mid \theta_{0}}
\end{array}\right. \\
& \geq 2\left\{\begin{array}{l}
P_{\min }\left(\theta_{0}-\xi, \theta_{0}\right) \\
P_{\min }\left(\theta_{0}, \theta_{0}+\xi\right)
\end{array}\right.
\end{aligned}
$$

where $P_{\epsilon_{1}}$ and $P_{\epsilon_{2}}$ denote the probabilities of error of the nearest decision rule

$$
\hat{H}=\left\{\begin{array}{l}
H_{1} \\
H_{2}
\end{array} \text { if }\left|\tilde{\Theta}-\left\{\Theta \mid H_{1}\right\}\right| \lessgtr\left|\tilde{\Theta}-\left\{\Theta \mid H_{2}\right\}\right|\right.
$$

of the two-hypothesis decision problems (the decision problem in (73) is illustrated in Fig. 5):

$$
\begin{aligned}
& H= \begin{cases}H_{1}: \Theta=\theta_{0}-\xi & P_{H_{1}}=0.5 \\
H_{2}: \Theta=\theta_{0} & P_{H_{2}}=0.5\end{cases} \\
& H= \begin{cases}H_{1}: \Theta=\theta_{0} & P_{H_{1}}=0.5 \\
H_{2}: \Theta=\theta_{0}+\xi & P_{H_{2}}=0.5\end{cases}
\end{aligned}
$$

and $P_{\min }\left(\theta_{0}-\xi, \theta_{0}\right)$ and $P_{\min }\left(\theta_{0}, \theta_{0}+\xi\right)$ the minimum probabilities of error obtained by the optimum decision rule based on the likelihood ratio test ([36], pp. 30):

$$
\hat{H}=\left\{\begin{array}{l}
H_{1} \\
H_{2}
\end{array} \text { if } \Lambda\left(\Theta \mid H_{1}\right)-\Lambda\left(\Theta \mid H_{2}\right) \gtrless \ln \frac{P_{H_{2}}}{P_{H_{1}}}\right.
$$

with $\Lambda(\theta)$ denoting the log-likelihood function in (2). The probability of error of an arbitrary detector $\hat{H}$ is given by

$$
P_{e}=P_{H_{1}} P_{\hat{H}=H_{2} \mid H_{1}}+P_{H_{2}} P_{\hat{H}=H_{1} \mid H_{2}} .
$$

${ }^{8}$ The obtained bounds are "approximate" due to this assumption; the assumption is valid when $\theta$ is not very close to the extremities of $D_{\Theta}$. 


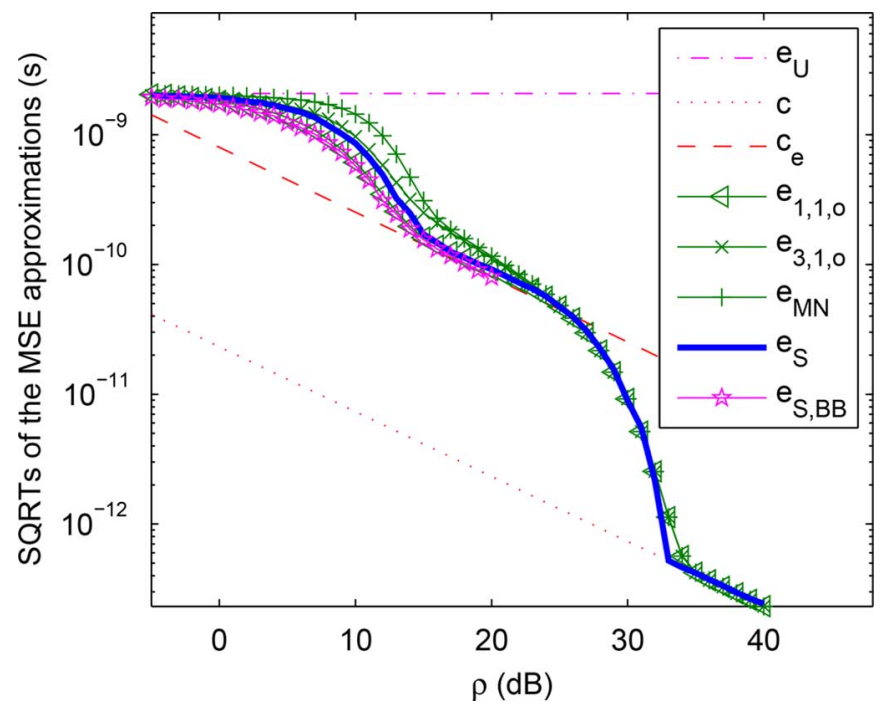

Fig. 8. Passband: SQRTs of the max. MSE $e_{U}$, the CRLB $c$, the ECRLB $c_{e}$, the MSEAs $e_{1,1, o}, e_{3,1, o}$ and $e_{M N}$, and the simulated MSEs of the passband $e_{S}$ and baseband $e_{S, B B}$ pulses, w.r.t. the SNR.

From (68) and (70) we obtain the following ALBs:

$$
\begin{aligned}
& z_{1}=\int_{0}^{\epsilon_{1}} \xi P_{\min }\left(\theta_{0}-\xi, \theta_{0}\right) d \xi \\
& z_{2}=\int_{0}^{\epsilon_{2}} \xi P_{\min }\left(\theta_{0}, \theta_{0}+\xi\right) d \xi
\end{aligned}
$$

where $\epsilon_{1}=\min \left\{\theta_{0}-\Theta_{1}, 2\left(\Theta_{2}-\theta_{0}\right)\right\}$ and $\epsilon_{2}=$ $\min \left\{\Theta_{2}-\theta_{0}, 2\left(\theta_{0}-\Theta_{1}\right)\right\}$. The integration limits are set to $\epsilon_{1}$ and $\epsilon_{2}$ to make the two hypotheses in (72) and (73) fall inside $D_{\Theta}$. As $P_{|\epsilon|>\frac{\xi}{2} \mid \theta_{0}}$ is a decreasing function, tighter bounds can be obtained by filling the valleys of $P_{\min }\left(\theta_{0}-\xi, \theta_{0}\right)$ and $P_{\min }\left(\theta_{0}, \theta_{0}+\xi\right)$ (as proposed by Bellini and Tartara in [4]):

$$
\begin{aligned}
& b_{1}=\int_{0}^{\epsilon_{1}} \xi V\left\{P_{\min }\left(\theta_{0}-\xi, \theta_{0}\right)\right\} d \xi \\
& b_{2}=\int_{0}^{\epsilon_{2}} \xi V\left\{P_{\min }\left(\theta_{0}, \theta_{0}+\xi\right)\right\} d \xi
\end{aligned}
$$

where $V\{f(\xi)\}=\max \{f(\zeta \geq \xi)\}$ denotes the valley-filling function. When $P_{\min }\left(\theta, \theta^{\prime}\right)$ is a function of $\theta^{\prime}-\theta$ (e.g., TOA estimation) we can write the bounds in (76)-(79) as $(i=1,2)$ :

$$
\begin{aligned}
z_{i} & =\int_{0}^{\epsilon_{i}} \xi P_{\min }(\xi) d \xi \\
b_{i} & =\int_{0}^{\epsilon_{i}} \xi V\left\{P_{\min }(\xi)\right\} d \xi .
\end{aligned}
$$

If $\theta_{0}-\Theta_{1}>\Theta_{2}-\theta_{0}$, then $\epsilon_{1}>\epsilon_{2}$; hence, $z_{1}$ and $b_{1}$ become tighter than $z_{2}$ and $b_{2}$, respectively. From (2), (28), (74) and (75) we can write the minimum probability of error as

$$
\begin{aligned}
P_{\min }\left(\theta, \theta^{\prime}\right) & =0.5\left[P_{\Lambda\left(\theta^{\prime}\right)>\Lambda(\theta) \mid \Theta=\theta}+P_{\Lambda(\theta)>\Lambda\left(\theta^{\prime}\right) \mid \Theta=\theta^{\prime}}\right] \\
& =0.5\left[\left.P\left(\theta^{\prime}, \theta\right)\right|_{\Theta=\theta}+\left.P\left(\theta, \theta^{\prime}\right)\right|_{\Theta=\theta^{\prime}}\right] \\
& =Q\left(\sqrt{\frac{\rho}{2}\left[1-R\left(\theta, \theta^{\prime}\right)\right]}\right) .
\end{aligned}
$$

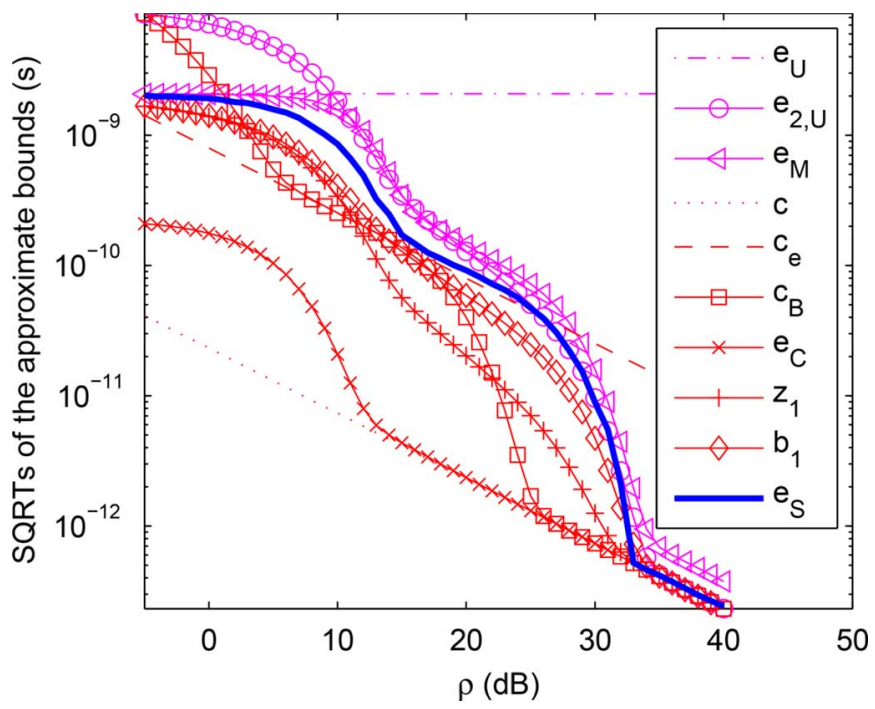

Fig. 9. Passband: SQRTs of the max. MSE $e_{U}$, the AUBs $e_{2, U}$ and $e_{M}$, the $\operatorname{CRLB} c$, the ECRLB $c_{e}$, the BLB $c_{B}$, the ALBs $e_{C}, z_{1}$ and $b_{1}$, and the simulated MSE $e_{S}$, w.r.t. the SNR.

There are two main differences between our bounds (deterministic) and the Bayesian ones: i) with the former we integrate along the error only whereas with the latter we integrate along the error and the a priori distribution of $\Theta$ (e.g., see (14) in [21]); ii) all hypotheses (e.g., $\Theta=\theta_{0}$ and $\Theta=\theta_{0}+\xi$ in (73)) are possible in the Bayesian case thanks to the a priori distribution whereas only one hypothesis $\left(\Theta=\theta_{0}\right)$ is possible in the deterministic case. So in order to utilize the minimum probability of error we have approximated $P_{\epsilon<-\frac{\xi}{2} \mid \theta_{0}}$ in (69) by $P_{\epsilon<-\frac{\xi}{2} \mid \theta_{0}+\xi}$ (see Fig. 5).

In this section we have two main contributions. The first one is the $\mathrm{ALB} e_{C}$ whereas the second one is the deterministic ZZLB family. These bounds can from now on be used as benchmarks in deterministic parameter estimation (like the CRLB) where it is not rigorous to use Bayesian bounds. Even though the derivation of $e_{c}$ was a bit complex, the final expression is now ready to be utilized.

\section{NumericAl ReSUltS AND DisCUSSION}

In this section we discuss some numerical results about the derived MSEAs, AUB, and ALBs. We consider TOA estimation using baseband and passband pulses. Let $T_{w}=2 \mathrm{~ns}, f_{c}=6.85$ GHz, $\Theta=0$ and $D_{\Theta}=[-2,1.5] T_{w}$. With the baseband (resp. passband) pulse we consider 9 equal duration intervals (resp. we consider an interval around each of the 48 local maxima). Let

$e_{i, j, x}=P_{0}^{(i)} \sigma_{0,0}^{2}+\sum_{n=n_{1}, n \neq 0}^{n_{N}} P_{n}^{(i)}\left[\left(\Theta-\mu_{n, j, x}\right)^{2}+\sigma_{n, j, x}^{2}\right]$

be the MSEA based on (22) and using the interval probability approximation $P_{n}^{(i)}(i \in\{1,2,3\}$, see (26), (27), (29)) and interval mean and variance approximations $\mu_{n, j, x}$ and $\sigma_{n, j, x}^{2}$ $((j, x)=U$ in (30), (31), and $(j, x) \in\{1,2\} \times\{c, o\}$ in (41)-(44), (49)-(52)). 


\section{A. Baseband Pulse}

Consider first the baseband pulse. In Fig. 6 we show the SQRTs of the maximum MSE $e_{U}$ in (19), the CRLB $c$ in (8), five MSEAs: $e_{1, U}, e_{1,1, c}, e_{1,2, c}, e_{3,1, c}$ in (83) and $e_{M N}$ in (60), and the MSE $e_{S}$ obtained by simulation based on 10000 trials, versus the SNR. In Fig. 7 we show the SQRTs of $e_{U}$, two AUBs: $e_{2, U}$ in (83) and $e_{M}$ in (57), $c$, the BLB $c_{B}$ in (18), two ALBs: $e_{C}$ in (67) and $z_{1}$ in (80) (equal to $b_{1}$ in (81) because a non-oscillating $\mathrm{ACR})$, and $e_{S}$.

We can see from $e_{S}$ that, as cleared up in Section I, the SNR axis can be divided into three regions: 1) the a priori region where $e_{U}$ is achieved, 2) the threshold region and 3) the asymptotic region where $c$ is achieved. We define the a priori and asymptotic thresholds by [7]:

$$
\begin{aligned}
& \rho_{p r}=\rho: e(\rho)=\alpha_{p r} e_{U} \\
& \rho_{a s}=\rho: e(\rho)=\alpha_{a s} c .
\end{aligned}
$$

We take $\alpha_{p r}=0.5$ and $\alpha_{p r}=1.1$. From $e_{S}$, we have $\rho_{p r}=4$ $\mathrm{dB}$ and $\rho_{a s}=16 \mathrm{~dB}$. Thresholds are defined in literature w.r.t. two magnitudes at least: i) the achieved MSE [7], [9], [21] like in our case (which is the most reliable because the main concern in estimation is to minimize the MSE) and ii) the probability of non-ambiguity [15], [37] (for simplicity reasons). Note that the RMSE achieved in the a priori region increases with the width of the a priori domain as can be seen from (19). This explains why the RMSE is relatively small at low SNRs (1.9 ns at -5 $\mathrm{dB}$ ) in our numerical example; in fact, the considered $D_{\Theta}$ is relatively narrow $\left(\Theta_{2}-\Theta_{1}\right.$ is 3.5 times the pulse width).

The MSEAs $e_{1, U}, e_{1,1, c}, e_{1,2, c}, e_{3,1, c}$ obtained from the MIE (Section IV) are very accurate and follow $e_{S}$ closely; $e_{1,1, c}$ is more accurate than $e_{3,1, c}$ which slightly overestimates $e_{S}$ because $e_{1,1, c}$ uses the probability approximation $P_{n}^{(1)}$ in (26) that considers all testpoints during the computation of the probability, whereas $e_{3,1, c}$ uses the approximation $P_{n}^{(3)}$ in (29) based on the probability UB $P_{n}^{(2)}$ in (27) that only considers the 0 th and the $n$th testpoints; $e_{1,1, c}$ is more accurate than $e_{1, U}$ which slightly overestimates $e_{S}$, and than $e_{1,2, c}$ which slightly underestimates it, because $e_{1,1, c}$ uses the variance approximation $\sigma_{n, 1, c}^{2}$ in (42) obtained from the first order Taylor series expansion of noise, whereas $e_{1, U}$ uses $\sigma_{n, U}^{2}$ in (31) assuming the MLE uniformly distributed in $D_{n}$ (overestimation of the noise), and $e_{1,2, c}$ uses $\sigma_{n, 2, c}^{2}$ in (44) neglecting the noise. The MSEA $e_{M N}$ proposed in Section V.A based on our probability approximation $P_{n}^{(3)}$ is very accurate as well.

The AUB $e_{2, U}$ proposed in [1] is very tight and converges to the asymptotic region simultaneously with $e_{S}$. However, it is less tight in the a priori and threshold regions because it uses the probability UB $P_{n}^{(2)}$ which is not very tight in these regions (see Fig. 3). Moreover, $e_{2, U} \rightarrow \infty$ when $N \rightarrow \infty$. The AUB $e_{M}$ (Section V.A) is very tight. However, it converges to 2.68 times the CRLB at high SNRs. This fact was discussed in Section V.A and also solved in Section V.B by proposing $e_{M N}$ (examined above). Nevertheless, $e_{M}$ can be used to compute the asymptotic threshold accurately because it converges to its own asymptotic regime simultaneously with $e_{S}$.
Both the BLB $c_{B}$ and the ALB $e_{C}$ (Section VI.A) outperform the CRLB. Unlike the passband case considered below, $e_{C}$ outperforms the BLB. The ALB $z_{1}$ (Section VI.B) is very tight and converges to the CRLB simultaneously with $e_{S}$.

\section{B. Passband Pulse}

Consider now the passband pulse. In Fig. 8 we show the SQRTs of the maximum MSE $e_{U}$, the CRLB $c$, the ECRLB $c_{e}$ in (15) (equal to CRLB of the baseband pulse), three MSEAs: $e_{1,1, o}$ and $e_{3,1, o}$ in (83) and $e_{M N}$ in (60), and the MSEs obtained by simulation for both the passband $e_{S}$ and the baseband $e_{S, B B}$ pulses. In Fig. 9 we show the SQRTs of $e_{U}$, two AUBs: $e_{2, U}$ in (83) and $e_{M}$ in (57), $c, c_{e}$, the BLB $c_{B}$, three ALBs: $e_{C}$ in (67), $z_{1}$ in (80) and $b_{1}$ in (81), and $e_{S}$.

By observing $e_{S}$, we identify five regions: 1) the a priori region, 2) the a priori-ambiguity transition region, 3) the ambiguity region where the ECRLB is achieved, 4) the ambiguity-asymptotic transition region and 5) the asymptotic region. We define the begin-ambiguity and end-ambiguity thresholds marking the ambiguity region by [7]

$$
\begin{aligned}
& \rho_{a m 1}=\rho: e(\rho)=\alpha_{a m 1} c_{e} \\
& \rho_{a m 2}=\rho: e(\rho)=\alpha_{a m 2} c_{e} .
\end{aligned}
$$

We take $\alpha_{a m 1}=2$ and $\alpha_{a m 2}=0.5$. From $e_{S}$ we have $\rho_{p r}=7$ $\mathrm{dB}, \rho_{a m 1}=15 \mathrm{~dB}, \rho_{a m 2}=28 \mathrm{~dB}$ and $\rho_{a s}=33 \mathrm{~dB}$.

The MSEAs $e_{1,1, o} ; e_{3,1, o}$ (Section IV) and $e_{M N}$ (Section V.B) are highly accurate and follow $e_{S}$ closely.

The AUB $e_{2, U}[1]$ is very tight beyond the a priori region. The AUB $e_{M}$ (Section V.A) is very tight. However, it converges to 1.75 times the CRLB in the asymptotic region.

The BLB $c_{B}$ detects the ambiguity and asymptotic regions much below the true ones; consequently, it does not determine accurately the thresholds $\left(\rho_{a m 1}=5 \mathrm{~dB}, \rho_{a m 2}=20 \mathrm{~dB}\right.$ and $\rho_{a s}=26 \mathrm{~dB}$ instead of 15,28 and $\left.33 \mathrm{~dB}\right)$. The ALB $e_{C}$ (Section VI.A) outperforms the CRLB, but is outperformed by the BLB (unlike the baseband case). The ALB $z_{1}$ (Section VI.B) is very tight, but $b_{1}$ (Section VI.B) is tighter thanks to the valleyfilling function. They both can calculate accurately the asymptotic threshold and to detect roughly the ambiguity region.

Let us compare the MSEs $e_{S, B B}$ and $e_{S}$ achieved by the baseband and passband pulses (Fig. 8). Both pulses approximately achieve the same MSE below the end-ambiguity threshold of the passband pulse $\left(\rho_{a m 2}=28 \mathrm{~dB}\right)$ and achieve the ECRLB between the begin-ambiguity and end-ambiguity thresholds. The MSE achieved with the baseband pulse is slightly smaller than that achieved with the passband pulse because with the former the estimates spread in continuous manner along the ACR whereas with the latter they spread around the local maxima. The asymptotic threshold of the baseband pulse (16 $\mathrm{dB})$ is approximately equal to the begin-ambiguity threshold of the passband pulse $(15 \mathrm{~dB})$. Above the end-ambiguity threshold, the MSE of the passband pulse rapidly converges to the CRLB while that of the baseband one remains equal to the ECRLB.

To summarize we can say that for a given nonlinear estimation problem with an oscillating ACR, the MSE achieved by the ACR below the end-ambiguity threshold is the same as that achieved by its envelope. Between the begin-ambiguity 
and end-ambiguity thresholds, the achieved MSE is equal to the ECRLB. Above the latter threshold, the MSE achieved by the ACR converges to the CRLB whereas that achieved by its envelope remains equal to the ECRLB.

\section{CONCLUSION}

We have considered nonlinear estimation of scalar deterministic parameters and investigated the threshold and ambiguity phenomena. The MIE is employed to approximate the statistics of the MLE. The obtained MSEAs are highly accurate and follow the true MSE closely. A very tight AUB is proposed as well. An ALB tighter than the CRLB is derived using the second order Taylor series expansion of noise. The principle of binary detection is utilized to compute some ALBs which are very tight.

\section{APPENDIX A \\ CuRvatures of THE ACR AND OF ITS ENVElope}

In this Appendix we prove (14). From (11) and (13) we can write the FT of the complex envelope $e_{R_{s}}(\theta, \Theta)$ as

$$
\mathcal{F}_{e_{R_{s}}}(f)=2 \mathcal{F}_{R_{s}}^{+}\left[f+f_{c}(\Theta)\right]
$$

where $x^{+}(f)=\left\{\begin{array}{cc}x(f) & f>0 \\ 0 & f \leq 0\end{array}\right.$. Form (13) we can write

$$
\begin{array}{r}
\ddot{R}_{s}(\theta, \Theta)=\Re\left\{e ^ { j 2 \pi ( \theta - \Theta ) f _ { c } ( \Theta ) } \left[j 4 \pi f_{c}(\Theta) \dot{e}_{R_{s}}(\theta, \Theta)\right.\right. \\
\left.\left.+\ddot{e}_{R_{s}}(\theta, \Theta)-4 \pi^{2} f_{c}^{2}(\Theta) e_{R_{s}}(\theta, \Theta)\right]\right\}
\end{array}
$$

As from (13) $\Re\left\{e_{R_{s}}(\Theta, \Theta)\right\}=R_{s}(\Theta, \Theta)=E_{s}$, (89) gives

$$
\begin{aligned}
\ddot{R}_{s}(\Theta, \Theta)=\Re\left\{\ddot{e}_{R_{s}}(\Theta, \Theta)\right\}-4 \pi^{2} f_{c}^{2}(\Theta) E_{s} \\
+4 \pi f_{c}(\Theta) \Re\left\{j \dot{e}_{R_{s}}(\Theta, \Theta)\right\} .
\end{aligned}
$$

To prove (14) from (90) we must prove that $\Re\left\{j \dot{e}_{R_{s}}(\Theta, \Theta)\right\}$ is null. Using (88) and the inverse FT, we can write

$$
\begin{aligned}
\dot{e}_{R_{s}}(\theta, \Theta) & =\int_{-\infty}^{+\infty} j 2 \pi f \mathcal{F}_{e_{R_{s}}}(f) e^{j 2 \pi f(\theta-\Theta)} d f \\
& =\int_{-\infty}^{+\infty} j 4 \pi f \mathcal{F}_{R_{s}}^{+}\left[f+f_{c}(\Theta)\right] e^{j 2 \pi f(\theta-\Theta)} d f \\
& =\int_{-\infty}^{+\infty} j 4 \pi\left[f-f_{c}(\Theta)\right] \mathcal{F}_{R_{s}}^{+}(f) e^{j 2 \pi\left[f-f_{c}(\Theta)\right](\theta-\Theta)} d f \\
& =\int_{0}^{+\infty} j 4 \pi\left[f-f_{c}(\Theta)\right] \mathcal{F}_{R_{s}}(f) e^{j 2 \pi\left[f-f_{c}(\Theta)\right](\theta-\Theta)} d f
\end{aligned}
$$

so $\dot{e}_{R_{s}}(\Theta, \Theta)=\int_{0}^{+\infty} j 4 \pi\left[f-f_{c}(\Theta)\right] \mathcal{F}_{R_{s}}(f) d f$. Using (12) and the last equation, $\Re\left\{j \dot{e}_{R_{s}}(\Theta, \Theta)\right\}$ becomes

$\Re\left\{j \dot{e}_{R_{s}}(\Theta, \Theta)\right\}=-\int_{0}^{+\infty} 4 \pi\left[f-f_{c}(\Theta)\right] \Re\left\{\mathcal{F}_{R_{s}}(f)\right\} d f=0$.

Hence, (14) is proved.

\section{ACKNOWLEDGMENT}

The authors would like to thank Prof. Alan Genz for his help in the probability numerical computation.

\section{REFERENCES}

[1] R. McAulay and D. Sakrison, "A PPM/PM hybrid modulation system," IEEE Trans. Commun. Technol., vol. 17, no. 4, pp. 458-469, Aug. 1969.

[2] J. Ziv and M. Zakai, "Some lower bounds on signal parameter estimation," IEEE Trans. Inf. Theory, vol. 15, no. 3, pp. 386-391, May 1969.

[3] L. Seidman, "Performance limitations and error calculations for parameter estimation," Proc. IEEE, vol. 58, no. 5, pp. 644-652, May 1970.

[4] S. Bellini and G. Tartara, "Bounds on error in signal parameter estimation," IEEE Trans. Commun., vol. 22, no. 3, pp. 340-342, Mar. 1974.

[5] S.-K. Chow and P. Schultheiss, "Delay estimation using narrow-band processes," IEEE Trans. Acoust., Speech, Signal Process., vol. 29, no. 3, pp. 478-484, June 1981.

[6] A. Weiss and E. Weinstein, "Fundamental limitations in passive time delay estimation-Part I: Narrow-band systems," IEEE Trans. Acoust., Speech, Signal Process., vol. 31, no. 2, pp. 472-486, Apr. 1983.

[7] E. Weinstein and A. Weiss, "Fundamental limitations in passive timedelay estimation-Part II: Wide-band systems," IEEE Trans. Acoust., Speech, Signal Process., vol. 32, no. 5, pp. 1064-1078, Oct. 1984.

[8] A. Zeira and P. Schultheiss, "Realizable lower bounds for time delay estimation," IEEE Trans. Signal Process., vol. 41, no. 11, pp. 3102-3113, Nov. 1993.

[9] A. Zeira and P. Schultheiss, "Realizable lower bounds for time delay estimation. 2. Threshold phenomena," IEEE Trans. Signal Process. vol. 42, no. 5, pp. 1001-1007, May 1994.

[10] B. Sadler and R. Kozick, "A survey of time delay estimation performance bounds," in Proc. 4th IEEE Workshop Sensor Array, Multichannel Process., Jul. 2006, pp. 282-288.

[11] B. Sadler, L. Huang, and Z. Xu, "Ziv-Zakai time delay estimation bound for ultra-wideband signals," in Proc. IEEE Int. Conf. Acoust., Speech, Signal Process. (ICASSP), Apr. 2007, vol. 3, pp. III-549-III-552.

[12] S. Zafer, S. Gezici, and I. Guvenc, Ultra-Wideband Positioning Systems: Theoretical Limits, Ranging Algorithms, and Protocols. Cambridge, U.K.: Cambridge Univ. Press, 2008.

[13] A. Renaux, P. Forster, E. Chaumette, and P. Larzabal, "On the high-SNR conditional maximum-likelihood estimator full statistical characterization," IEEE Trans. Signal Process., vol. 54, no. 12, pp. 4840-4843, Dec. 2006.

[14] A. Renaux, P. Forster, E. Boyer, and P. Larzabal, "Unconditional maximum likelihood performance at finite number of samples and high signal-to-noise ratio," IEEE Trans. Signal Process., vol. 55, no. 5, pp. 2358-2364, May 2007.

[15] C. Richmond, "Capon algorithm mean-squared error threshold SNR prediction and probability of resolution," IEEE Trans. Signal Process., vol. 53, no. 8, pp. 2748-2764, Aug. 2005.

[16] A. Renaux, "Contribution à l'analyse des performances d'estimation en traitement statistique du signal," Ph.D. dissertation, ENS CACHAN, Cachan, France, 2006.

[17] L. Seidman, "An upper bound on average estimation error in nonlinear systems," IEEE Trans. Inf. Theory, vol. 14, no. 2, pp. 243-250, Mar. 1968.

[18] , H. L. Van Trees and K. L. Bell, Eds., Bayesian Bounds for Parameter Estimation and Nonlinear Filtering/Tracking. New York, NY, USA: Wiley-IEEE Press, 2007.

[19] D. Chazan, M. Zakai, and J. Ziv, "Improved lower bounds on signal parameter estimation," IEEE Trans. Inf. Theory, vol. 21, no. 1, pp. 90-93, Jan. 1975.

[20] E. Weinstein, "Relations between Belini-Tartara, Chazan-Zakai-Ziv, and Wax-Ziv lower bounds," IEEE Trans. Inf. Theory, vol. 34, no. 2, pp. 342-343, Mar. 1988.

[21] K. Bell, Y. Steinberg, Y. Ephraim, and H. Van Trees, "Extended ZivZakai lower bound for vector parameter estimation," IEEE Trans. Inf. Theory, vol. 43, no. 2, pp. 624-637, Mar. 1997.

[22] I. Reuven and H. Messer, "A Barankin-type lower bound on the estimation error of a hybrid parameter vector," IEEE Trans. Inf. Theory, vol. 43, no. 3, pp. 1084-1093, May 1997.

[23] S. Kay, Fundamentals of Statistical Signal Processing Estimation Theory. Englewood Cliffs, NJ, USA: Prentice-Hall, 1993.

[24] E. W. Barankin, "Locally best unbiased estimators," Ann. Math. Statist., vol. 20, pp. 477-501, Dec. 1949.

[25] R. McAulay and L. Seidman, "A useful form of the Barankin lower bound and its application to PPM threshold analysis," IEEE Trans. Inf. Theory, vol. 15, no. 2, pp. 273-279, Mar. 1969.

[26] R. McAulay and E. Hofstetter, "Barankin bounds on parameter estimation," IEEE Trans. Inf. Theory, vol. 17, no. 6, pp. 669-676, Nov. 1971. 
[27] L. Seidman, "The performance of a PPM/PM hybrid modulation system," IEEE Trans. Commun. Technol., vol. 18, no. 5, pp. 697-698, Oct. 1970.

[28] P. Swerling, "Parameter estimation for waveforms in additive Gaussian noise," J. Soc. Ind. Appl. Math., vol. 7, no. 2, pp. 152-166, June 1959.

[29] L. Knockaert, "The Barankin bound and threshold behavior in frequency estimation," IEEE Trans. Signal Process., vol. 45, no. 9, pp. 2398-2401, Sep. 1997.

[30] D. Dardari, C.-C. Chong, and M. Win, "Improved lower bounds on time-of-arrival estimation error in realistic UWB channels," in Proc. IEEE Int. Conf. Ultra-Wideband (ICUWB), Sep. 2006, pp. 531-537.

[31] B. Sadler, L. Huang, and Z. Xu, "Ziv-Zakai time delay estimation bound for ultra-wideband signals," in Proc. IEEE Int. Conf. Acoust., Speech, Signal Process. (ICASSP), Apr. 2007, vol. 3, pp. III-549-III-552.

[32] R. Kozick and B. Sadler, "Bounds and algorithms for time delay estimation on parallel, flat fading channels," in Proc. IEEE Int. Conf. Acoust., Speech, Signal Process., (ICASSP), Apr. 2008, pp. 2413-2416.

[33] D. Dardari, C.-C. Chong, and M. Win, "Threshold-based time-of-arrival estimators in UWB dense multipath channels," IEEE Trans. Commun., vol. 56, no. 8, pp. 1366-1378, Aug. 2008.

[34] D. Dardari, A. Conti, U. Ferner, A. Giorgetti, and M. Win, "Ranging with ultrawide bandwidth signals in multipath environments," Proc. IEEE, vol. 97, no. 2, pp. 404-426, Feb. 2009.

[35] D. Dardari and M. Win, "Ziv-Zakai bound on time-of-arrival estimation with statistical channel knowledge at the receiver," in Proc. IEEE Int. Conf. Ultra-Wideband (ICUWB), Sep. 2009, pp. 624-629.

[36] J. M. Wozencraft and I. M. Jacobs, Principles of Communication Engineering. New York, NY, USA: Wiley, 1965.

[37] H. L. Van Trees, Detection, Estimation, and Modulation Theory, Part I. New York, NY, USA: Wiley, 1968.

[38] P. M. Woodward, Probability and Information Theory With Applications to Radar. New York, NY, USA: McGraw-Hill, 1955.

[39] V. A. Kotelnikov, The Theory of Optimum Noise Immunity. New York, NY, USA: McGraw-Hill, 1959.

[40] D. Rife and R. Boorstyn, "Single tone parameter estimation from discrete-time observations," IEEE Trans. Inf. Theory, vol. 20, no. 5, pp. 591-598, Sep. 1974.

[41] L. Najjar-Atallah, P. Larzabal, and P. Forster, "Threshold region determination of ML estimation in known phase data-aided frequency synchronization," IEEE Signal Process. Lett., vol. 12, no. 9, pp. 605-608, Sep. 2005.

[42] E. Boyer, P. Forster, and P. Larzabal, "Nonasymptotic statistical performance of beamforming for deterministic signals," IEEE Signal Process. Lett., vol. 11, no. 1, pp. 20-22, Jan. 2004.

[43] F. Athley, "Threshold region performance of maximum likelihood direction of arrival estimators," IEEE Trans. Signal Process., vol. 53, no. 4, pp. 1359-1373, Apr. 2005.

[44] C. Richmond, "Mean-squared error and threshold SNR prediction of maximum-likelihood signal parameter estimation with estimated colored noise covariances," IEEE Trans. Inf. Theory, vol. 52, no. 5, pp. 2146-2164, May 2006.

[45] A. Mallat, S. Gezici, D. Dardari, and L. Vandendorpe, "Statistics of the MLE and approximate upper and lower bounds-Part II: Threshold computation and optimal pulse design for TOA estimation," IEEE Trans. Signal Process., vol. 62, no. 21, pp. 5677-5689, 2014.

[46] A. Mallat, J. Louveaux, and L. Vandendorpe, "UWB based positioning in multipath channels: CRBs for AOA and for hybrid TOA-AOA based methods," in Proc. IEEE Int. Conf. Commun. (ICC), Jun. 2007, pp. $5775-5780$.

[47] , M. I. Skolnik, Ed., Radar Handbook. New York, NY, USA: McGraw-Hill, 1970.

[48] Revision of Part 15 of the Commission Rules Regarding Ultra-Wideband Transmission Systems, FCC 02-48, Federal Communications Commission (FCC), Apr. 2002.

[49] A. Mallat, P. Gerard, M. Drouguet, F. Keshmiri, C. Oestges, C. Craeye, D. Flandre, and L. Vandendorpe, "Testbed for IR-UWB based ranging and positioning: Experimental performance and comparison to CRLBs," in Proc. 5th IEEE Int. Symp. Wireless Pervasive Comput. (ISWPC), May 2010, pp. 163-168.

[50] A. Genz, "Numerical computation of multivariate normal probabilities," J. Comput. Graph. Statist., vol. 1, no. 2, pp. 141-149, June 1992.

[51] A. Genz, "On a number-theoretical integration method," Aequationes Mathematicae, vol. 8, no. 3, pp. 304-311, Oct. 1972.

[52] A. Genz, "Randomization of number theoretic methods for multiple integration," SIAM J. Numer. Anal., vol. 13, no. 6, pp. 904-914, Dec. 1976.
[53] D. Nuyens and R. Cools, "Fast component-by-component construction, a reprise for different kernels," in Monte-Carlo and Quasi-Monte Carlo Methods, H. Niederreiter and D. Talay, Eds. New York, NY, USA: Springer-Verlag, 2004, pp. 371-385.

[54] H. I. Jacobson, "The maximum variance of restricted unimodal distributions," Annu. Math. Statist., vol. 40, no. 5, pp. 1746-1752, Oct. 1969.

[55] S. W. Dharmadhikari and K. Joag-Dev, "Upper bounds for the variances of certain random variables," Commun. Statist. Theory Methods, vol. 18, no. 9, pp. 3235-3247, 1989.

[56] G. Marsaglia, "Ratios of normal variables and ratios of sums of uniform variables," J. Amer. Statist. Assoc., vol. 60, no. 309, pp. 193-204, Mar. 1965.

[57] G. Marsaglia, "Ratios of normal variables," J. Statist. Softw., vol. 16, no. 4, May 2006.

[58] D. V. Hinkley, "On the ratio of two correlated normal random variable," Biometrika, vol. 56, no. 3, pp. 635-639, Dec. 1969.

[59] E. Cinlar, Introduction to Stochastic Process. Englewood Cliffs, NJ, USA: Prentice Hall, 1975.

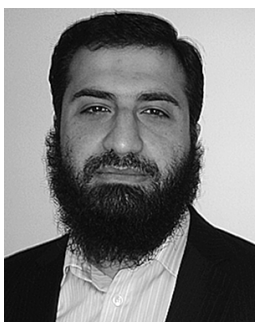

Achraf Mallat (S'06-M'14) received his B.Sc. degree in electrical and electronics engineering from the Lebanese University, Faculty of Engineering, Branch I, Tripoli, Lebanon, in 2002, his M.Sc. degree in signal, telecommunications, image and radar from the Université de Rennes 1, Rennes, France, in 2004, and his Ph.D. degree in engineering sciences from the Université Catholique de Louvain (UCL), Louvain-la-Neuve, Belgium, in 2013. From September 2005 to August 2013, he was a Research/Teaching Assistant at the Louvain School of Engineering, UCL. Since September 2013, he has been a Postdoctoral Researcher with the Institute for Information and Communication Technologies, Electronics and Applied Mathematics, UCL. His current research interests are in the areas of signal processing and parameter estimation, and, in particular, in ultra-wideband (UWB) based positioning, and automotive radar systems.

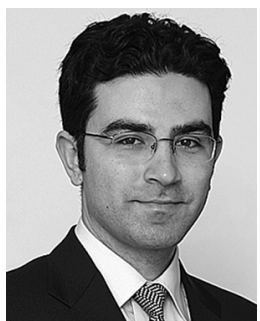

Sinan Gezici (S'03-M'06-SM'11) received the B.S. degree from Bilkent University, Turkey, in 2001, and the Ph.D. degree in electrical engineering from Princeton University in 2006. From 2006 to 2007, he worked at Mitsubishi Electric Research Laboratories, Cambridge, MA, USA. Since 2007, he has been with the Department of Electrical and Electronics Engineering at Bilkent University, where he is currently an Associate Professor. Dr. Gezici's research interests are in the areas of detection and estimation theory, wireless communications, and localization systems. Among his publications in these areas is the book Ultrawideband Positioning Systems: Theoretical Limits, Ranging Algorithms, and Protocols (Cambridge University Press, 2008). Dr. Gezici is an associate editor for the IEEE TRANSACTIONS ON COMMUNICATIONS, the IEEE WIRELESS COMMUNICATIONS LETTERS, and the Journal of Communications and Networks.

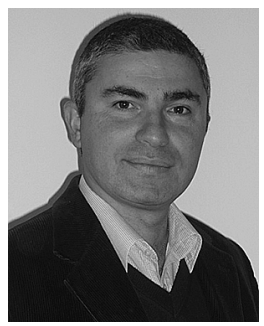

Davide Dardari (M'95-SM'07) received the Laurea degree in electronic engineering (summa cum laude) and the Ph.D. degree in electronic engineering and computer science from the University of Bologna, Italy, in 1993 and 1998, respectively. $\mathrm{He}$ is an Associate Professor at the University of Bologna at Cesena, Italy, where he participates with WiLAB (Wireless Communications Laboratory). Since 2005, he has been a Research Affiliate at Massachusetts Institute of Technology (MIT), Cambridge, USA. He is also Research Affiliate at IEIIT/CNR (National Research Council) and CNIT (Consorzio Nazionale Interuniversitario per le Telecomunicazioni). He published more than 150 technical papers and played several important roles in various National and European Projects. He is coauthor of the books Wireless Sensor and Actuator Networks: Enabling Technologies, Information Processing and Protocol Design (Elsevier, 2008) and 
Satellite and Terrestrial Radio Positioning Techniques-A Signal Processing Perspective (Elsevier, 2011). His interests are on ultra-wide bandwidth (UWB) systems, ranging and localization techniques, distributed signal processing, as well as wireless sensor networks. He received the IEEE Aerospace and Electronic Systems Society's M. Barry Carlton Award (2011) and the IEEE Communications Society Fred W. Ellersick Prize (2012). Prof. Dardari is Senior Member of the IEEE, where he was the Chair for the Radio Communications Committee of the IEEE Communication Society. He was Co-General Chair of the 2011 IEEE International Conference on Ultra-Wideband and co-organizer of the first and second IEEE International Workshop on Advances in Network Localization and Navigation (ANLN) - ICC 2013-2014. He was also Co-Chair of the Wireless Communications Symposium of the 2007 IEEE International Conference on Communications, and Co-Chair of the 2006 IEEE International Conference on Ultra-Wideband. He served as Lead Editor for the EURASIP Journal on Advances in Signal Processing (Special Issue on Cooperative Localization in Wireless Ad Hoc and Sensor Networks), Guest Editor for ProceEdings of IEEE (Special Issue on UWB Technology \& Emerging Applications), for the Physical Communication Journal (ELSEVIER) (Special Issue on Advances in UWB Wireless Communications), and for the IEEE TRANSActions on Vehicular Technology (Special Session on Indoor Localization, Tracking, and Mapping With Heterogeneous Technologies). He served as an Editor for the IEEE TRANSACTIONS ON WIRELESS COMMUNICATIONS from 2006 to 2012.

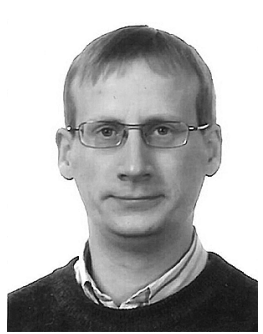

Christophe Craeye (M'98-SM'11) was born in Belgium in 1971. He received the Electrical Engineer and Bachelier in philosophy degrees and $\mathrm{Ph} . \mathrm{D}$. degree in applied sciences from the Université Catholique de Louvain (UCL), Louvain-La-Neuve, Belgium, in 1994 and 1998, respectively. From 1994 to 1999 , he was a Teaching Assistant with UCL and carried out research on the radar signature of the sea surface perturbed by rain, in collaboration with NASA and ESA. From 1999 to 2001, he was a Post-Doc Researcher with the Eindhoven University of Technology, Eindhoven, The Netherlands. His research there was related to wideband phased arrays devoted to the Square Kilometer Array radio telescope. In this framework, he also was with the University of Massachusetts, Amherst, MA, USA, in the Fall of 1999, and worked with the Netherlands Institute for Research in Astronomy, Dwingeloo, The Netherlands, in 2001. In 2002, he started an antenna research activity at the Universite Catholique de Louvain, where he is now a Professor. He was with the Astrophysics and Detectors Group, University of Cambridge, Cambridge, U.K., from January to August 2011. His research interests are finite antenna arrays, wideband antennas, small antennas, metamaterials, and numerical methods for fields in periodic media, with applications to communication, sensing and positioning systems. His research is funded by Région Wallonne, European Commission, FNRS, and
UCL. Prof. Craeye served as an Associate Editor of the IEEE TRANSACTIONS ON ANTENNAS AND PROPAGATION from 2004 to 2010. He is now an Associate Editor for the IEEE ANTENNAS AND WiRELESS PROPAGATION LETTERS. In 2009, he received the 2005-2008 Georges Vanderlinden Prize from the Belgian Royal Academy of Sciences.

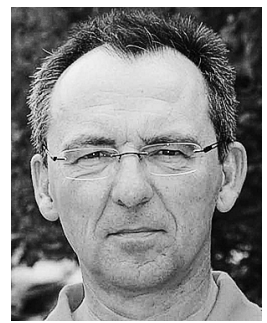

Luc Vandendorpe (F'06) was born in Mouscron, Belgium, in 1962. He received the electrical engineering degree (summa cum laude) and the Ph.D. degree from the Université Catholique de Louvain (UCL), Louvain-la-Neuve, Belgium, in 1985 and 1991, respectively. Since 1985 , he has been with the Communications and Remote Sensing Laboratory, UCL, where he first worked in the field of bit rate reduction techniques for video coding. In 1992, he was a Visiting Scientist and Research Fellow with the Telecommunications and Traffic Control Systems Group, Delft Technical University, The Netherlands, where he worked on spread spectrum techniques for personal communications systems. From October 1992 to August 1997, he was Senior Research Associate of the Belgian NSF at UCL, and an Invited Assistant Professor. Presently, he is Professor and Head of the Institute for Information and Communication Technologies, Electronics and Applied Mathematics. His current interest is in digital communication systems and more precisely resource allocation for OFDM(A)-based multicell systems, MIMO and distributed MIMO, sensor networks, turbo based communications systems, physical layer security, and UWB-based positioning. Dr. Vandendorpe was a co-recipient of the Biennal Alcatel-Bell Award from the Belgian NSF for a contribution in the field of image coding in 1990. In 2000, he was a co-recipient (with J. Louveaux and F. Deryck) of the Biennal Siemens Award from the Belgian NSF for a contribution about filter bank based multicarrier transmission. In 2004, he was a co-winner (with J. Czyz) of the Face Authentication Competition, FAC 2004. $\mathrm{He}$ is or has been a TPC member for numerous IEEE conferences (VTC Fall, Globecom Communications Theory Symposium, SPAWC, ICC) and for the Turbo Symposium. He was Co-Technical Chair (with P. Duhamel) for IEEE ICASSP 2006. He was an editor of the IEEE TRANSACTIONS ON COMMUNICATIONS FOR SYNCHRONIZATION AND EQUALIZATION between 2000 and 2002, Associate Editor of the IEEE TRANSACTIONS ON WIRELESS COMMUNICATIONS between 2003 and 2005, and Associate Editor of the IEEE Transactions on Signal PROCESSING between 2004 and 2006. He was Chair of the IEEE Benelux joint chapter on Communications and Vehicular Technology between 1999 and 2003. He was an elected member of the Signal Processing for Communications Committee between 2000 and 2005, and between 2009 and 2011, and an elected member of the Sensor Array and multichannel Signal Processing Committee of the Signal Processing Society between 2006 and 2008. He is the Editor-in-Chief for the EURASIP Journal on Wireless Communications and Networking. 